2013

\title{
Understanding the Rhythm of Breathing: So Near, Yet So Far
}

Jack L. Feldman

Christopher A. Del Negro

William \& Mary, cadeln@wm.edu

Paul A. Gray

Follow this and additional works at: https://scholarworks.wm.edu/aspubs

\section{Recommended Citation}

Feldman, J. L., Del Negro, C. A., \& Gray, P. A. (2013). Understanding the rhythm of breathing: so near, yet so far. Annual review of physiology, 75, 423-452.

This Article is brought to you for free and open access by the Arts and Sciences at W\&M ScholarWorks. It has been accepted for inclusion in Arts \& Sciences Articles by an authorized administrator of W\&M ScholarWorks. For more information, please contact scholarworks@wm.edu. 


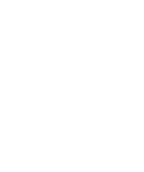
ANNUAL Further
REVIEWS

Click here for quick links to Annual Reviews content online, including:

- Other articles in this volume

- Top cited articles

- Top downloaded articles

- Our comprehensive search

\section{Understanding the Rhythm of Breathing: So Near, Yet So Far}

\section{Jack L. Feldman, ${ }^{1}$ Christopher A. Del Negro, ${ }^{2}$ and Paul A. Gray ${ }^{3}$}

\author{
${ }^{1}$ Department of Neurobiology, David Geffen School of Medicine, University of California, \\ Los Angeles, California 90095-1763; email: feldman@ucla.edu \\ ${ }^{2}$ Department of Applied Science, The College of William \& Mary, Williamsburg, Virginia \\ 23187-8795; email: cadeln@ $@_{\text {wm.edu }}$ \\ ${ }^{3}$ Department of Anatomy and Neurobiology, Washington University School of Medicine, St. \\ Louis, Missouri 63110-1093; email: pgray@pcg.wustl.edu
}

Annu. Rev. Physiol. 2013. 75:423-52

First published online as a Review in Advance on October 29, 2012

The Annual Review of Physiology is online at http://physiol.annualreviews.org

This article's doi:

10.1146/annurev-physiol-040510-130049

Copyright (c) 2013 by Annual Reviews.

All rights reserved

\section{Keywords}

preBötzinger Complex, retrotrapezoid nucleus, parafacial respiratory group, central pattern generation, brain stem

\begin{abstract}
Breathing is an essential behavior that presents a unique opportunity to understand how the nervous system functions normally, how it balances inherent robustness with a highly regulated lability, how it adapts to both rapidly and slowly changing conditions, and how particular dysfunctions result in disease. We focus on recent advancements related to two essential sites for respiratory rhythmogenesis: (a) the preBötzinger Complex (preBötC) as the site for the generation of inspiratory rhythm and $(b)$ the retrotrapezoid nucleus/parafacial respiratory group (RTN/pFRG) as the site for the generation of active expiration.
\end{abstract}




\section{INTRODUCTION}

Understanding the mechanisms leading from DNA to molecules to neurons to networks to behavior is a major goal for neuroscience but is largely out of reach for many fundamental and interesting behaviors. The neural control of breathing may be a rare exception, presenting a unique opportunity to understand how the nervous system functions normally, how it balances inherent robustness with a highly regulated lability, how it adapts to both rapidly and slowly changing conditions, and how particular dysfunctions result in disease. Why can we assert this? First and foremost, the functions of breathing are clearly definable, starting with its regulatory role of maintaining blood (and brain) $\mathrm{O}_{2}, \mathrm{CO}_{2}$, and $\mathrm{pH}$; failure is not an option. Breathing is also an essential component of many vocal and emotive behaviors, e.g., crying, laughing, singing, and sniffing, and must be coordinated with vital behaviors such as suckling and swallowing, even at birth. Second, the regulated variables- $\mathrm{O}_{2}, \mathrm{CO}_{2}$, and $\mathrm{pH}$ (and temperature in nonprimate mammals) — are continuous and are readily and precisely quantifiable, as are ventilation itself along with the underlying rhythmic motor activity, i.e., respiratory muscle EMGs. Third, we breathe all the time, except for short breaks such as those during breath holding (which can be especially long in diving or hibernating mammals) or sleep apnea. Mammals (including humans) breathe in all behavioral states, e.g., in sleep-wake, in rest-exercise, in panic/fear-calm, during anesthesia, and even following loss of cortical function or decerebration. Moreover, essential aspects of the neural mechanisms driving breathing, including rhythmicity, are present at levels of reduction down to a medullary slice. Fourth, the relevant circuits exhibit a remarkable combination of extraordinary reliability, starting ex utero with the first air breath (intermittent breathing movements actually start in utero during the third trimester) and continuing for as many as $\sim 10^{9}$ breaths, as well as considerable lability, responding rapidly (in less than one second) and with considerable precision over an order of magnitude in metabolic demand for $\mathrm{O}_{2}\left(\sim 0.25\right.$ to $\sim 5$ liters of $\mathrm{O}_{2}$ per minute). Breathing does indeed persist! Finally, breathing is genetically determined to work at birth, with a well-defined developmental program underlying a neuroanatomical organization with apparent segregation of function; i.e., rhythmogenesis is separate from motor pattern (burst shapes and coordination) generation. Importantly, single-human-gene mutations can affect breathing, and several neurodegenerative disorders compromise breathing by direct effects on brain stem respiratory circuits.

Figure 1 presents a broad overview of the central pattern generator for breathing. We distinguish rhythmogenesis from the more complex process of pattern generation, which is the production of precisely coordinated and timed motor nerve burst patterns across a broad array of muscles pumping the lung or controlling airflow resistance. A substantial portion of the nervous system that controls breathing movements is not involved in rhythmogenesis but instead transforms rhythmic signals into the appropriate pattern of muscle contraction. These critical but nonrhythmogenic structures include motor and premotor neurons. In addition, there are sensory neurons, including mechanoreceptors and chemoreceptors. Here we focus on recent advancements related to two essential sites for rhythmogenesis: (a) the preBötzinger Complex (preBötC) in the generation of inspiratory rhythm and $(b)$ the retrotrapezoid nucleus/parafacial respiratory group (RTN/pFRG) in the generation of active expiration. We first discuss their function, anatomy, and physiology, providing a foundation for us to then discuss their developmental genetics.

Caveat lector: Making sense of the literature on respiratory rhythmogenesis is challenging. The ultimate goal is to understand normal and pathological breathing in humans. However, very little experimentation relevant to rhythmogenesis can be done in humans, in whom the location and relatively small size of the relevant neural structures in the brain stem and the prevalence of 


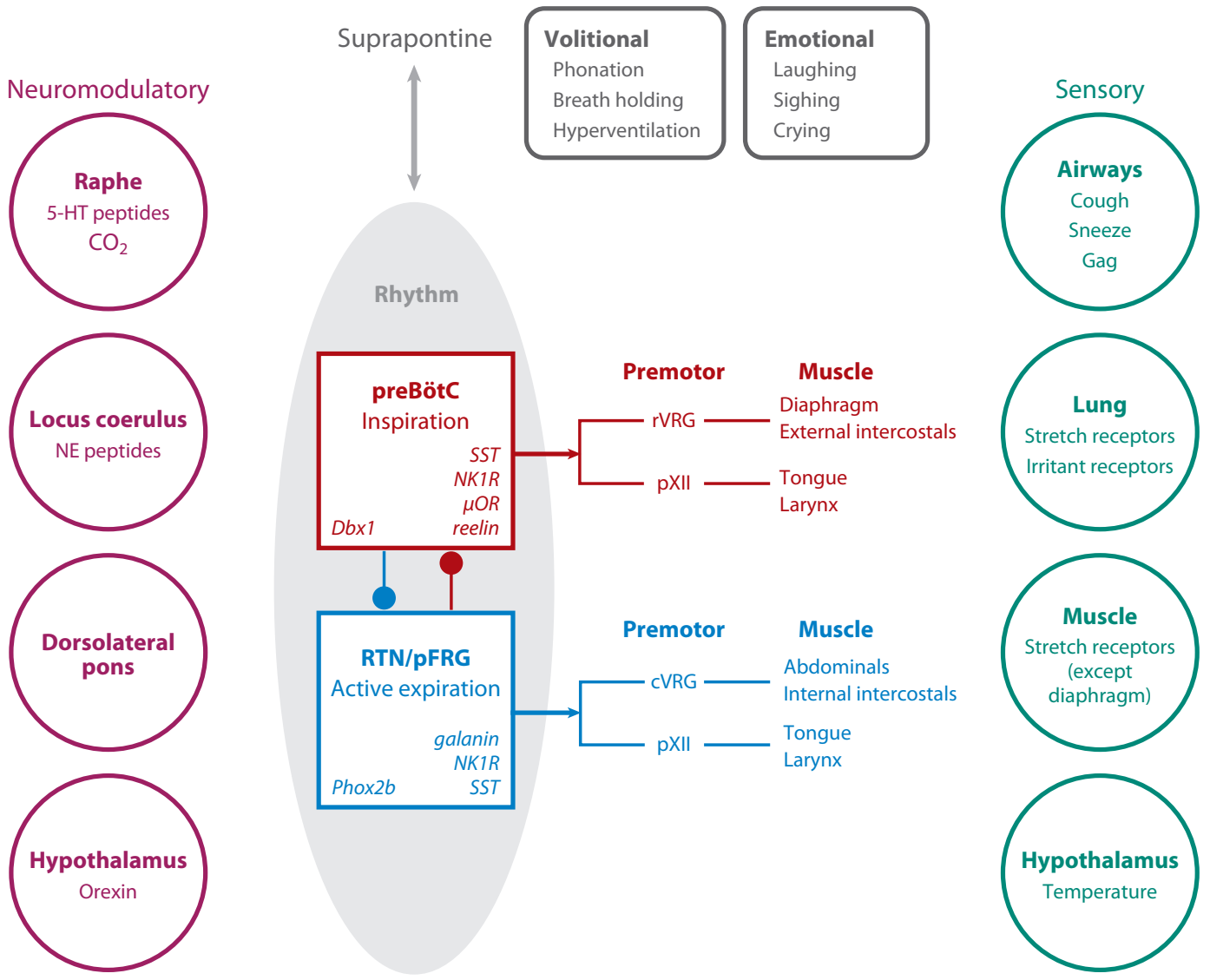

Figure 1

Overview of the central pattern generator for breathing. Core rhythm-generating circuits appear to have two distinct brain stem oscillators: the endogenously active preBötzinger Complex (preBötC) (red box) and the conditionally active retrotrapezoid nucleus/parafacial respiratory group (RTN/pFRG) (blue box). The preBötC drives inspiratory activity by projections to various premotor populations [rostral ventral respiratory group (rVRG), parahypoglossal (pXII)] that in turn project both to inspiratory muscles that pump air, e.g., the diaphragm and external intercostals, and to inspiratory muscles that modulate airflow resistance, e.g., laryngeal and tongue muscles. The RTN/pFRG has a similar functional path to expiratory muscles. preBötC and RTN/pFRG progenitors and neurons express distinct transcription factors $(D b x 1$ or Phox $2 b)$ and other genes (right; italics). Numerous neuromodulatory (left), suprapontine (top), and sensory (right) influences are shown. (Left) Neuromodulatory influences. Respiratory pattern is highly labile. When you go from quiet sitting to slow walking, your $\mathrm{O}_{2}$ consumption increases approximately threefold, and if your ventilation does not increase rapidly, you will probably pass out within $100 \mathrm{~m}$. Peptides, serotonin, norepinephrine, and other endogenous neuromodulators_originating in projections from, for example, the raphe, locus coerulus, and hypothalamus—can affect rhythmogenesis. These actions are essential for normal regulation and may go awry in diseases affecting breathing. The dorsolateral pons, aka pontine respiratory group, including the Kölliker-Fuse and parabrachial nuclei, is also an important modulatory source. (Top) Suprapontine inputs are related to volition and emotion. (Right) Sensory inputs are essential for the proper regulation of blood gases and for mechanical adjustments related to posture, body mechanics, and likely metabolic efficiency.

heartbeat- and breathing-induced movements prevent invasive techniques and render noninvasive neuroimaging techniques difficult (1). All nonhuman in vivo experimental models are studied while breathing or studied in vitro or in situ while generating breathing-related neural activity, with breathing rhythm and pattern highly dependent on the details of the experimental preparation. In more or less intact mammals, breathing is markedly affected by species (and even by strain), 
body temperature, blood gas level, anesthetic (with different anesthetics having different actions on breathing), paralysis, decerebration, integrity of peripheral nerves such as the carotid sinus and vagus nerves (which can be intact or cut), and sleep-wake state. In in situ preparations, the blood-brain barrier is compromised. In rhythmic slice preparations, slight variations in the rostral or caudal borders can markedly affect the pattern of motor output, as does the composition of the bathing fluid, which can vary considerably in $\mathrm{K}^{+}(3-9 \mathrm{mM})$ and $\mathrm{Ca}^{2+}(0.8-2.5 \mathrm{mM})$ between different laboratories (2-7). Unfortunately, we tend to pick and choose among results that align with our biases, often ignoring confounds that temper or invalidate the comparisons, and to dismiss those results that do not agree.

The terminology for breathing patterns, once well defined and essential for straightforward comparison of experimental results (8), has devolved. During eupnea, commonly understood to mean quiet breathing or resting respiration in fully intact mammals, neural output to respiratory muscles is regular: Rhythmic bursts of motor activity to the diaphragm and external intercostal muscles drive inspiratory airflow, and expiratory airflow results from passive recoil of the lung and rib cage. The term eupnea has now been stretched to include the respiratory motor nerve patterns in hypothermic, aortic perfused, vagotomized, blood-brain barrier-compromised rodent preparations that generate respiratory motor output patterns that are quite different from those in intact rodents at rest (9). Further confusing the issue is the addition of the term fictive, such as fictive eupnea, to describe the pattern of XII nerve activity in rhythmically active slice preparations (10). As another example, gasping in its long-standing definition described the very stereotyped last breaths in anoxic mammals just before they would asphyxiate, absent a rescue of some type. However, gasping now refers to various respiratory motor nerve patterns in highly reduced preparations (1012), often to dismiss such activity as irrelevant to understanding mechanisms underlying eupnea, however defined; we strongly disagree with the shift in the use of the term gasping. Experimental limitations and discontinuity of definitions and terminology notwithstanding, the advances in the past six years provide considerable new insights into the sites and mechanisms underlying breathing.

\section{PREBÖTZINGER COMPLEX AND INSPIRATION}

There is a broad consensus (with plenty of quibbling over details) for the answer to the ageold question, "Where does breathing originate?" The paradigms underlying investigation of the neural control of breathing underwent a shift with the establishment of in vitro experimental models relevant to breathing $(5,13)$. Such models led to the identification of the preBötC and the hypothesis that it was the kernel for respiratory rhythmogenesis $(5,14)$. In previous reviews (1517), we discussed key experiments that tested this hypothesis in vivo. To better understand recent work, we note three findings. (a) The preBötC is both necessary and sufficient for the generation of inspiratory motor, i.e., hypoglossal nerve (XIIn), output in in vitro rodent slice preparations $(5,14)$. (b) Juvenile rats in vivo with brain stem transection just rostral to the preBötC continue to generate rhythmic inspiratory-dominated breathing patterns (18), as do in situ heart-brain stem preparations (19). (c) Neurotoxic lesioning of a peptide receptor-defined subset of preBötC neurons results in ataxic breathing in otherwise intact rats. Approximately 300 glutamatergic preBötC neurons per side express the neurokinin-1 receptor (NK1R), for which substance $\mathrm{P}$ (SP) is the ligand. A toxin, saporin, conjugated to SP (SP-SAP) selectively kills, over a period of days, neurons that express NK1R. Injection of SP-SAP into the preBötC in intact adult rats induces, after several days, a disturbed breathing pattern during sleep; with more complete lesions, ataxic breathing occurs during wakefulness (20), and apneas (the complete cessation of breathing) occur during sleep (21). 

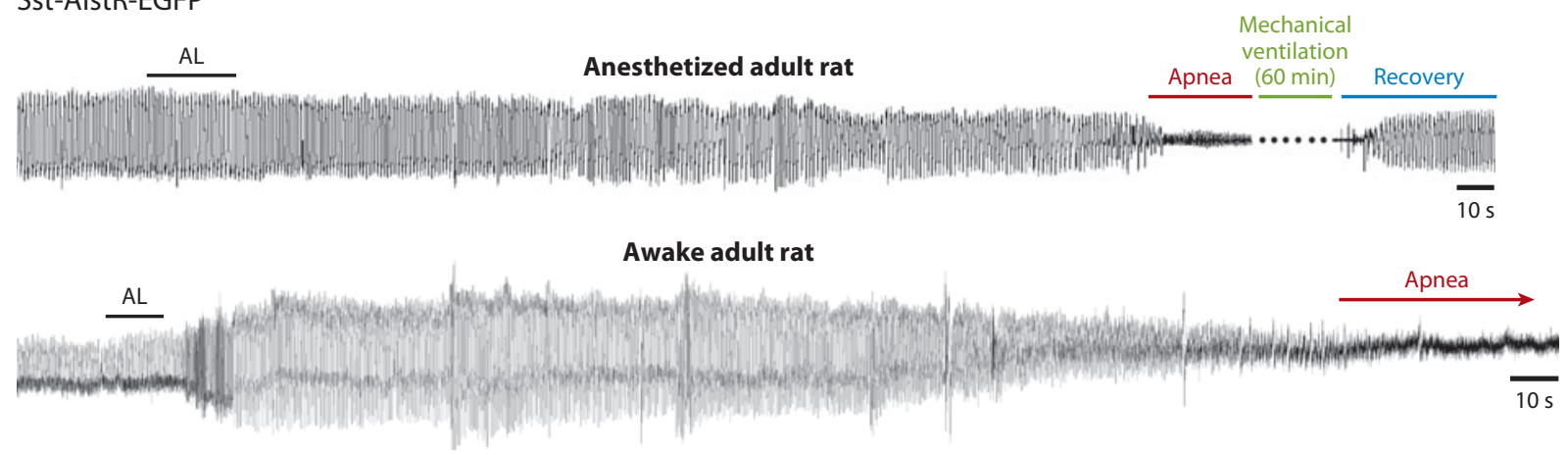

Figure 2

The preBötC is essential for breathing in adult rats. Rapid silencing of allatostatin receptor (AlstR)-expressing preBötC somatostatin (Sst) neurons induces persistent apnea (lasting $>45 \mathrm{~min}$ with mechanical ventilation) in anesthetized (top) or awake (bottom) adult rats. Neurons were transfected by local, bilateral injection into the preBötC of an AAV2 virus expressing AlstR under a Sst promoter. The traces are plethysmographic recordings. The allatostatin ligand (AL), when administered intracerebrocisternally, induces a gradual decline of frequency and tidal volume until apnea develops after several minutes. After $\sim 60 \mathrm{~min}$ of mechanical ventilation, the rats resume spontaneous breathing. EGFP denotes enhanced green fluorescent protein. From Reference 24.

\section{Further (Smoking-Gun) Evidence for the preBötC as the Kernel for Inspiration}

Saporin-lesioned rats survive, albeit with a markedly pathological ataxic breathing pattern (21), for up to 12 days, leaving the unlikely possibility that another site drives breathing under normal conditions. Severe hypoxia and hypercapnea caused by increasingly long and frequent apneas (20) can cause neuronal death. Moreover, slow neurodegeneration with saporin makes it difficult to separate the specific and immediate effects of the loss of preBötC $\mathrm{NK}_{1} \mathrm{R}^{+}$neurons from secondary effects, including plasticity and bystander death. To eliminate these confounding effects of slow degeneration, a largely overlapping subpopulation of glutamatergic preBötC neurons that express the neuropeptide somatostatin (Sst) $(\sim 300$ per side) $(22,23)$ can be transiently suppressed by transfection with the Drosophila allatostatin receptor (AlstR) and subsequent exposure to the allatostatin ligand (AL). AL does not appear to activate any mammalian receptors. Rat neurons made to express the AlstR hyperpolarize when exposed to AL. When AL is introduced into the brain stem of control adult rats, there is no effect on breathing. However, in anesthetized or awake adult rats whose preBötC Sst neurons express AlstR, AL rapidly induces a remarkably persistent and profound apnea that requires mechanical ventilation to prevent asphyxiation (Figure 2) (24). Implicit in this observation is that these rats generate no breathing movements; there are no volitional or emotive movements, sighs, or gasps. These compelling data substantiate the essential role for preBötC neurons in normal breathing, i.e., eupnea.

\section{Respiratory Rhythm Generation in the preBötC}

If understanding the neural control of breathing requires solving a series of problems, foremost is delineating the mechanism for rhythmogenesis. More generally, rhythms, oscillations, and periodic bursting of various sorts are central to almost all aspects of brain function, ranging from rhythmically patterned movements (25), to cortical and cerebellar rhythms associated with signal processing and state, to slower processes with daily or seasonal rhythms. Breathing presents a unique window into neural rhythmogenesis because of a convergence of its essential properties: robustness, lability, a localized and identified rhythm generator (preBötC) [a key piece of the 
puzzle lacking for locomotion or chewing (26)], and various levels of reduction in vitro that provide exceptional access to neurons and networks for imaging and electrophysiology while maintaining spontaneous breathing-related motor output.

We postulate that rhythm is generated at the core of the respiratory central pattern generator and that this core is not concerned primarily with details of the output pattern. Our view is that timing signals normally originate in the preBötC and are broadcast to the rest of the network, perhaps by a subset of glutamatergic neurons with widespread projections (27) that transform the timing signals into appropriate patterns of muscle contraction (Figure 1). Adopting this point of view allows one to focus on the oscillation per se and on its neural origins. As mentioned above, the highly reduced slice preparation is experimentally optimal to elucidate such mechanisms. Our explicit assumption is that mechanisms gleaned in slices represent the foundation for rhythmogenic mechanisms in vivo. This point of view is opposite to assertions that rhythm generated in the slice is irrelevant to eupneic breathing in intact mammals (see below and References 11 and 19). In spite of all the inherent experimental advantages and the deceptive simplicity of rhythmogenesis, understanding rhythmogenesis in the preBötC in vitro is a tough problem.

Before the preBötC hypothesis $(5,14)$, proposals for rhythmogenesis focused on building balland-stick models consisting of populations of respiratory-modulated point/spherical neurons, i.e., neurons without dendrites, classified according to firing pattern with simple cellular properties, e.g., no voltage dependency, no ion selectivity, no metabotropic actions, and straightforward excitatory or inhibitory connections $(8,28-30)$. Once the intrinsic membrane properties of preBötC neurons were measured in vitro, these schematic models were elaborated. Currently, there are two major viewpoints for the mechanism of preBötC rhythm generation on the basis of the widely accepted finding that the preBötC rhythm is generated by glutamatergic interneurons $(31,32)$. Their distinction lies in whether the inspiratory burst initiates due to a small population of specialized pacemaker neurons (discussed below) or results from excitatory interactions among preBötC neurons that express synaptically triggered burst-generating conductances.

\section{Role of Glutamatergic Pacemaker Neurons in Respiratory Rhythm Generation}

We evaluated the evidence for and against possible roles of pacemaker neurons more than a decade ago in a previous Annual Review of Physiology review (16). These critiques, however, remain relevant to more recent work that pertains to the preBötC, as well as to pacemaker-driven models of locomotor and oral-motor rhythm generation (26,33-35). The heuristic power of the relatively straightforward pacemaker hypothesis may, in part, explain its persistence in the face of significant conflicting data. Over the past decade, some investigators have moderated their view, interpreting newer data as demonstrating that pacemakers and their underlying intrinsic conductances can contribute to rhythmogenesis but conceding that they are not essential (19, 36-38). Yet many authors assert that the preBötC rhythm is pacemaker driven and to interpret their data or build their models in that framework $(12,39-44)$.

preBötC rhythms in vitro continue without significant changes in frequency after blockade of chloride-mediated inhibition (45-48). This finding rules out mechanisms predicated on phase transitions that require conventional postsynaptic inhibition. The original observation catalyzed a search for and subsequent identification of preBötC neurons with bursting pacemaker properties that may best be defined by the ability to generate $(a)$ rhythmic membrane polarization absent rhythmic input and/or (b) ectopic bursts of rhythmic activity when depolarized (5, 49-51).

Bursting in preBötC neurons has two underlying ionic mechanisms. The first is voltage sensitive and depends on subthreshold activation of persistent $\mathrm{Na}^{+}$current $\left(I_{\mathrm{NaP}}\right)$ in neurons with sufficiently low leakage-like $\mathrm{K}^{+}$current $(38,51-53)$. $I_{\mathrm{NaP}}$-dependent bursting is present in a subset 
of preBötC neurons at least from birth as well as throughout the medulla postnatally; however, it is not a specialized property of preBötC neurons (52, q.v. 54, 55). The second bursting mechanism depends on a $\mathrm{Ca}^{2+}$-activated nonspecific cationic current $\left(I_{\mathrm{CAN}}\right)(50)$, whose activation mechanism in the absence of synaptic input depends on voltage-gated $\mathrm{Ca}^{2+}$ channels. Bursting of this type is less voltage sensitive (see figure $4 c$ in Reference 39; see also Reference 49) and emerges in a subset of preBötC neurons after postnatal day $4(\mathrm{P} 4)(42,49)$ with its relative prevalence influenced by neuromodulation $(12,39,41,56,57)$. Under most experimental conditions in slices from neonatal rodents, $\sim 5-25 \%$ of all preBötC inspiratory neurons (glutamatergic or glycinergic) exhibit bursting pacemaker properties $(38,42,49,58,59)$. However, the logic in the literature gets murky as one proceeds from the observation that pacemakers are present to conclusions that these pacemaker neurons generate the rhythm.

Arguments for the pacemaker hypothesis generally hinge on two observations. First, pacemaker neurons oscillate with a period and burst duration that match the duty cycle of the respiratory rhythm in vitro. Second, conditions that regulate the period and burst duration of synaptically isolated pacemaker neurons, e.g., high $\mathrm{K}^{+}$, neuromodulators, and ion channel agonists/antagonists, have similar effects on respiratory rhythm in vitro. These arguments assume that pacemaker neurons are glutamatergic/excitatory, which, alas, is not the case. In transgenic mice that express enhanced green fluorescent protein (EGFP) in glycinergic neurons, in which $\sim 50 \%$ of preBötC neurons fluoresce (60), $23 \%$ of inspiratory glycinergic preBötC neurons have voltage-dependent pacemaker properties (59).

Tests of the pacemaker hypothesis are not straightforward in execution or in interpretation. Antagonists of $I_{\mathrm{NaP}}$ or $I_{\mathrm{CAN}}$ such as riluzole or flufenamic acid invariably preclude intrinsic bursting in isolated pacemaker neurons but do not always perturb or stop the rhythm when bath applied or locally infused into the preBötC of slices or en bloc preparations or in situ (38, 42, 49, 61-63). Nevertheless, under the best of circumstances (which is likely never the case), rhythm cessation after drug exposure is a necessary but not sufficient condition if pacemakers are essential for rhythmogenesis. That is because $I_{\mathrm{NaP}}$ and $I_{\mathrm{CAN}}$ antagonists markedly affect other membrane properties and depress excitatory transmission in all neurons. Their net effect is a widespread decrease in neuronal excitability that is not limited to particular respiratory or even nonrespiratory nuclei (61, 64). Additionally, local infusion of an $I_{\mathrm{NaP}}$ antagonist into the midline nucleus raphe obscurus also stops the rhythm $(62,65)$. Therefore, rhythm cessation by drug application cannot be attributed to effects solely on preBötC neurons, and the results of pharmacological tests are at best equivocally in support of the pacemaker hypothesis. The conclusions that $I_{\mathrm{NaP}}$ pacemaker neurons drive gasping-like behavior in reduced in vitro and in situ preparations $(11,42)$ and that $I_{\mathrm{NaP}}$-dependent pacemaker activity drives spinal locomotor patterns $(34,66)$ are similarly unconvincing.

\section{Can Respiratory Rhythm Be Generated Without Pacemaker Neurons and Without Synaptic Inhibition?}

Previously $(16,17)$ we described a schematic group-pacemaker mechanism (7) that subsequently gave rise to explicit models for respiratory rhythm generation $(67,68)$. Before explaining how a group pacemaker works, we should note that there are few definitive data in support of the group-pacemaker model. It is at present a working hypothesis and framework for further testing. In the model, a fraction of glutamatergic preBötC neurons fire tonically at a low rate $(<1 \mathrm{~Hz})$ between inspiratory bursts. Percolating activity in this interconnected subset of respiratory neurons (69) increases via positive feedback. The part of the cycle in which positive feedback dominates other network constituents is properly considered the preinspiratory phase because it precedes the inspiratory burst by $300-400 \mathrm{~ms}$. According to the group-pacemaker hypothesis, recurrent 
excitation builds up during the preinspiratory phase and leads to a network-wide synchronous inspiratory burst phase. Consistent with this model, a ramp-like increase in the baseline membrane potential accompanied by low-rate spontaneous spiking during the preinspiratory phase is observed in so-called type 1 neurons, which are thought to be rhythmogenic on the basis of NK1 receptor expression (see above) and physiologically verified excitatory synaptic interconnections in the preBötC (Figure 3d) (69).

The termination of the inspiratory burst is still unresolved. Synaptic depression is a possible mechanism that would halt recurrent excitation (67). Burst termination may also involve activitydependent outward currents evoked by intense spiking, including $\mathrm{Na}^{+} / \mathrm{K}^{+}$ATPase electrogenic pump current, $\mathrm{Na}^{+}$-dependent $\mathrm{K}^{+}$current, and ATP-dependent $\mathrm{K}^{+}$current $(6,70-73)$.

\section{$I_{\text {CAN }}$, Burst Generation, and Dendritic Excitability}

Active dendritic membrane properties, in concert with intracellular signaling, can couple synaptic input to burst generation. Because inspiratory bursts are much larger in magnitude (and intraburst spiking is more intense: $20-100 \mathrm{~Hz}$ ) compared with temporal summation of excitatory postsynaptic potentials (EPSPs) and low-rate $(2-5-\mathrm{Hz})$ spiking during preinspiratory activity, the onset of the inspiratory burst reflects the recruitment of postsynaptic conductances that amplify synaptic drive but are unrelated to $I_{\mathrm{NaP}}(62)$. Morphology may play a role in synaptic amplification. Rall's analyses of dendritic properties (e.g., Reference 74), the advent of dendritic patch-clamp recordings, and new laser-imaging technologies demonstrate a wide array of excitable properties in dendrites that significantly influence synaptic integration, specifically the spatiotemporal summation of EPSPs (75). In preBötC neurons, the activation of AMPA receptors as well as of group I metabotropic glutamate receptors (mGluRs) evokes postsynaptic $\mathrm{Ca}^{2+}$ transients that in turn evoke $I_{\text {CAN }}$, either locally at the dendritic input site $(76,77)$ or via propagating $\mathrm{Ca}^{2+}$ waves (Figure $3 \boldsymbol{a}, \boldsymbol{b})(68)$. The net result is that, during the inspiratory burst phase, excitatory synaptic inputs evoke inward currents that promote robust inspiratory burst generation often recognized by depolarization block of spiking during the inspiratory phase (Figure $3 \boldsymbol{a}, \boldsymbol{b}, \boldsymbol{d}$ ). Somatic $\mathrm{Ca}^{2+}$ transients, in contrast, do not appear to play a critical role (78). The underlying ion channels appear to be members of the transient receptor potential (TRP) family, most likely TRPM4 and TRPM5 $(68,73,79)$ or possibly TRPC3 and TRPC7 (40). Inspiratory bursts with depolarization block are shown in a representative excitatory (glutamatergic) rhythmogenic preBötC neuron (rhythm-generating neurons are derived from precursors that express Dbx1; see below) in Figure $3 \boldsymbol{c}, \boldsymbol{d}$. The dendrites of $\mathrm{Dbx}^{+}$neurons are planar and radiate dorsoventrally $300-500 \mu \mathrm{m}$, often beyond the borders of the preBötC (Figure $3 \boldsymbol{c}, \boldsymbol{e}$ ). Dendritic excitability is critically important in understanding neuron computation in general (80). In a rhythmic central pattern generator, the ability to summate and synchronize excitatory inputs in support of periodic burst generation may be a function particularly well suited for dendrites, which we have just begun to exploit in order to elucidate key aspects of respiratory rhythmogenesis (81).

\section{Role of Inhibition in Rhythmogenesis and Pattern Formation}

Inhibition is an essential element that underlies rhythmic movements in invertebrates, in which most central-pattern-generator neurons are inhibitory (82). For locomotion in mammals, reciprocal inhibition was first proposed as the mechanism for the alternating rhythm around each joint $(83,84)$. Although this mechanism remains hypothetical, inhibition clearly underlies left-right limb alternations $(85,86)$. For breathing, postsynaptic inhibition is postulated, sometimes stipulated, as an essential element for rhythmogenesis (87-89). Inhibitory currents and potentials 

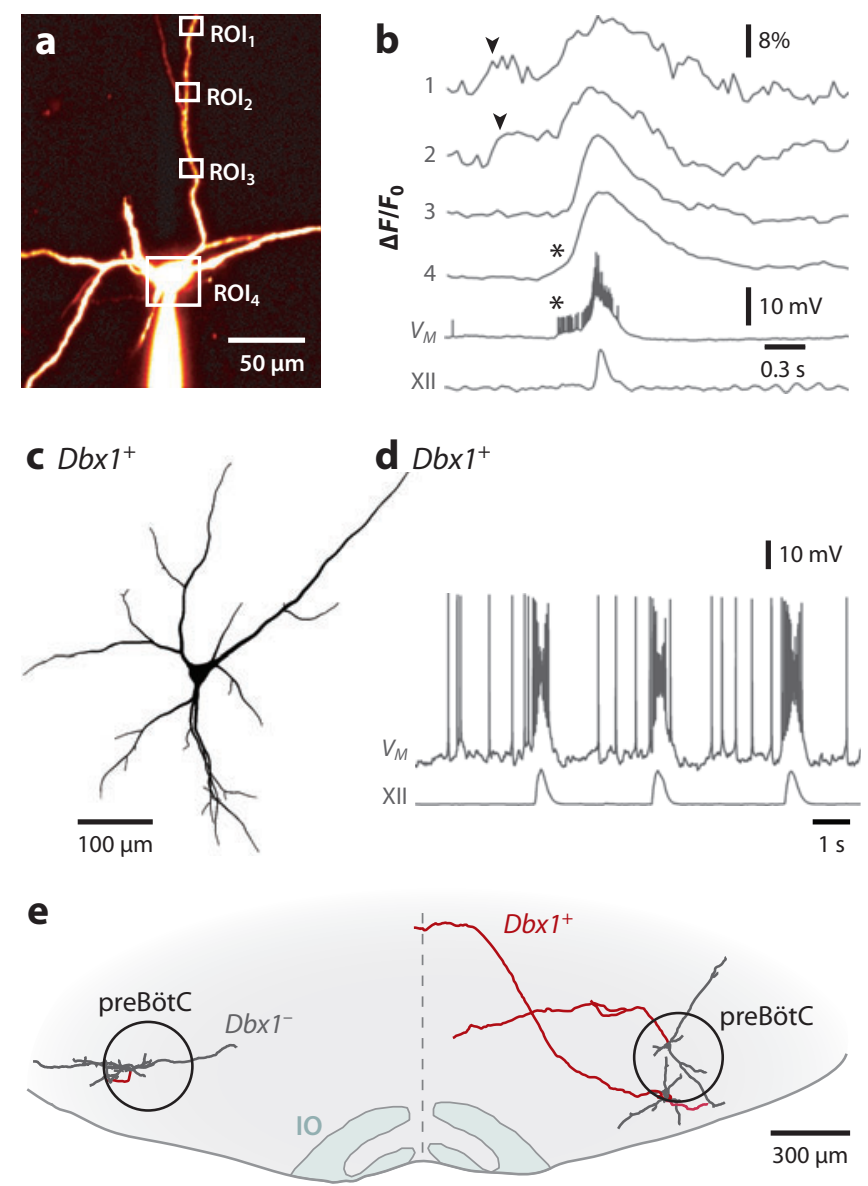

Figure 3

Inspiratory burst generation: the role of dendrites and the properties of $D b x 1^{+}$preBötC neurons. $(a, b)$ Dendritic two-photon $\mathrm{Ca}^{2+}$ imaging and somatic patch clamp. $(a)$ preBötC neuron filled with fluorescent dye from a somatic whole-cell recording. Numbered regions of interest (ROIs) correspond to the numbers in panel $b$. (b) Dendritic $\mathrm{Ca}^{2+}$ transients (arrowheads) precede somatic bursts and XII motor output. Asterisks indicate somatic spike-driven $\mathrm{Ca}^{2+}$ transients. From Reference 81. $(c, d)$ Morphology $(c)$ and physiology $(d)$ of a $D b x 1^{+}$preBötC neuron. Drive potentials of $\sim 25-\mathrm{mV}$ amplitude and depolarization block of spiking are indicative of $I_{\mathrm{CAN}}\left(\mathrm{Ca}^{2+}\right.$-activated nonspecific cationic current) activation during the inspiratory burst. (e) Transverse view of a mouse slice (ventral) showing two Dbx $1^{+}$neurons (right) and a $D b x 1^{-}$neuron $(l e f t)$ recorded and biocytin reconstructed. All three are inspiratory neurons. $D b x 1^{+}$neurons are commissural (axons in red). Abbreviations: IO, inferior olive; $V_{M}$, membrane voltage; XII, hypoglossal motor root.

are readily observed in respiratory-modulated brain stem neurons in intact mammals and are quite prominent when recorded under barbiturate anesthesia (30). The core mechanism for rhythm generation in one current, widely disseminated model is an inhibitory ring of three distinct neuronal populations of preBötC and BötC (just rostral to the preBötC and containing a high concentration of inhibitory respiratory-modulated neurons) neurons that sequentially inhibit each other and transform a presumptively tonically active subpopulation of excitatory preBötC neurons into an inspiratory-modulated one $(19,90)$. Block of synaptic inhibition throughout the entire neuraxis of a reduced in situ-perfused rat preparation produces apnea with tonic firing 
of preBötC and BötC neurons (19). Under certain pathological conditions, such as when the hindbrain is transected just rostral to preBötC in this in situ preparation, the inhibitory ring is presumably broken. According to this model, preBötC pacemaker neurons are released from tonic excitation and endogenously burst, driving a respiratory rhythm by a completely different mechanism. This presumptively pacemaker-driven rhythm is strikingly different from that presumably produced by the inhibitory ring in the following ways: (a) Inspiratory and expiratory outputs discharge synchronously, $(b)$ inspiratory bursts change shape from incrementing to decrementing, and $(c)$ respiratory frequency drops. According to the model, removing the inhibitory ring should produce apnea $(11,19,90)$. However, apnea apparently does not occur. In anesthetized, adult, spontaneously breathing, vagus-intact rats, effectively complete antagonism of $\mathrm{GABA}_{\mathrm{A}}$ and glycine receptors in the preBötC and BötC slows down the rhythm to that of a vagotomized rat; i.e., no apnea is induced (90a). This result suggests that postsynaptic inhibition within the preBötC (and the BötC) is not essential for the generation of respiratory rhythm, consistent with the group-pacemaker model. The primary role of inhibition appears to be in shaping the pattern of respiratory motor output and in assuring its stability, but not in the generation of rhythm per se.

\section{Opiates and the preBötC}

Opiates are a class of compounds that can depress breathing such that, at too high a concentration, asphyxiation occurs, as can happen when opiates are administered as an analgesic or are taken recreationally. $\mu$-Opioid receptors are present on preBötC neurons and when activated reduce their excitability (91). These preBötC $\mu$-opioid receptors appear to be responsible for the respiratory depressive effects of opiates (92). A functional antagonist of the $\mu$-opioid receptor-signaling pathway in preBötC neurons is the $5 \mathrm{HT}_{4}$ receptor, which does not appear to be present in pain pathways (93). In the adult rat, systemic administration of a $5 \mathrm{HT}_{4}$ agonist reverses the respiratory depressive effects of systemic administration of fentanyl (a potent $\mu$-opioid agonist) without a significant reduction in analgesia. More to the point, depression of breathing by systemic administration of fentanyl is fully reversed by focal injection into the preBötC of naloxone, a $\mu$-opioid antagonist (92). These observations provide further proof of the importance of the preBötC in breathing and suggest the potential of protocols to reduce the dangers of opiate analgesics.

\section{REDISCOVERY OF EXPIRATION}

Until recently, the paradigm for rhythmogenesis was that, regardless of the underlying mechanism, all phases of respiratory motor outflow originate from the same source(s). Thus, the preBötC was envisioned to sequentially parcel out signals to produce the alternating rhythm of inspiratory and expiratory movements. This does not appear to be the case. The diaphragm, an extraordinarily powerful muscle for inspiratory movements, is a defining characteristic of mammals. Breathing is the only mammalian behavior requiring the continuous movement of skeletal muscles; breathing consumes $\sim 7 \%$ of metabolic output at rest, and that number is much higher in pulmonary disease (94). In mammals, the most efficient pattern for rhythmic breathing at rest is active inspiration, which is generated by forces resulting from diaphragmatic and external intercostal muscle contraction acting to expand the lung, alternating with passive expiration due to the forces generated by the elastic recoil of the inspiratory muscles, lung, and rib cage. Modest increases in ventilation near resting values, such as during slow walking in humans, are typically accomplished by increasing inspiratory (tidal) volume, combined with decreasing expiratory duration, with expiration remaining (predominantly) passive (95). During exercise, e.g., when one chases prey or 
flees predators, there is a transition from passive to active expiration (96) due to the onset of contraction of the abdominal and internal intercostal muscles. In the past several years, there has been a significant paradigm shift, with the current view that rhythm originates from two distinct but coupled oscillatory populations with segregated function, i.e., one population for inspiration and one for expiration (Figure 1).

This shift began with the observation that both in vitro and in vivo, low-dose opioids can produce an occasional dropout of the inspiratory phase, i.e., quantal slowing (97), which requires structures rostral to the preBötC (18). This observation led to the hypothesis of an opiateinsensitive second oscillator that drives active expiration $(18,97)$. The location of this second oscillator was suggested by observations in in vitro en bloc preparations, in which a population of neurons rostral to the preBötC and mostly ventral to the facial nucleus (VIIn), dubbed the parafacial respiratory group (pFRG), was active prior to the onset of inspiratory activity (98). The pFRG overlaps with (and may even be identical to) the retrotrapezoid nucleus (RTN) (see below), which is postulated to be a site for central chemoreception for $\mathrm{CO}_{2}$ (99-101). Pending resolution as to their unique identity, we refer to this region as the RTN/pFRG $(15,102)$. The nonchemosensitive function of the RTN/pFRG was initially hypothesized to be the primary source of inspiratory rhythm (98). However, in juvenile rats generating active expiration, transection of the brain stem just caudal to the RTN/pFRG abolishes active expiration without much effect on inspiratory motor activity (18). The RTN/pFRG is postulated as a conditional oscillator for active expiration that is quiescent under certain conditions (18). Reduced preparations containing the RTN/pFRG but lacking the preBötC generate rhythmic output from VII motor roots under opioid inhibition (103), consistent with the picture of the RTN/pFRG as an independent oscillator. The absence of significant expiratory neuronal activity in the RTN/pFRG of normocapnic, normoxic, adult, anesthetized, mechanically ventilated rats $(104,105)$ reflects the absence of active expiration (17). Thus, stimulating the lateral RTN/pFRG via local disinhibition or photoactivation with channelrhodopsin transforms a resting breathing pattern with no active expiration into one with active expiration associated with the activation of late-expiratory RTN/pFRG neurons (Figure 4) (106). These results are consistent with the presence in the RTN/pFRG of an expiratory oscillator that when turned on promotes active expiration. Although elevated $\mathrm{CO}_{2}$ stimulates active expiration, elevated $\mathrm{CO}_{2}$ is not a prevalent stimulus in the normal life of most mammals. Rather, we suggest that descending signals related to exercise (107) or to a powerful emotional response, e.g., fear, are likely to turn on the RTN/pFRG oscillator to produce active expiration. Moreover, active expiration sometimes occurs in anesthetized mammals (108-110) or in highly reduced preparations (111-113), suggesting that multiple mechanisms may gate its activity.

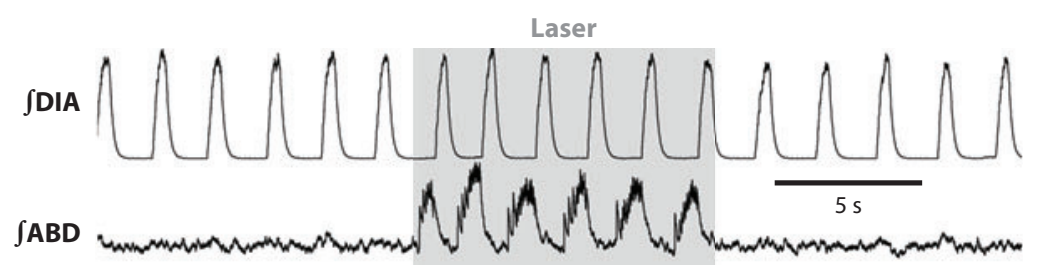

Figure 4

Passive expiration transformed into active expiration. The anesthetized adult rat has active inspiration, reflected in integrated diaphragm EMG (DIA), but passive expiration, reflected in tonic integrated abdominal EMG (ABD). Photoactivation of lateral RTN/pFRG neurons transfected with ChR2 (gray band) induces active expiration. 


\section{Expiration, Like Gaul, Is Divided into Three Parts}

Regardless of its origin, expiration has subphases. In particular, the transitions at the beginning and end of the expiratory phase are referred to as the postinspiratory (early-expiratory) and preinspiratory (late-expiratory) periods. In the postinspiratory period, following a sharp decline in phrenic nerve activity, inspiratory airflow stops and expiratory airflow begins. Just after this point, there is often a small, postinspiratory burst of phrenic nerve activity. This burst causes a lengthening contraction of the diaphragm that slows expiratory airflow. During the preinspiratory phase, $(a)$ in vitro, type 1 , putatively rhythmogenic preBötC neurons exhibit a ramp-like slow depolarization (Figure 3d (7), and (b) in vivo, stimuli that when delivered earlier in expiration affect expiratory duration $\left(T_{\mathrm{E}}\right)$ are ineffective, e.g., Reference 114 . Whether postinspiratory output constitutes a distinct phase of breathing rhythmogenesis (a three-phase model) $(19,90)$ or a motor subcomponent of the expiratory phase (a two-phase model) (18) is controversial. The timing of abdominal activity depends, in part, on the experimental condition; some experiments report purely preinspiratory activity $(108,110)$ or a mixture of pre- and postinspiratory activity $(18,111)$. Similar flexibility in motor patterns is present in reduced neonatal preparations $(4,113)$.

\section{RESPIRATORY CONNECTOME}

\section{preBötC Intrinsic Connections}

Regardless of the critical cellular properties, the connectivity among preBötC neurons must play an essential role in the stability and lability of the rhythm and perhaps in rhythmogenesis itself. This information has been challenging to obtain. Type 1 inspiratory preBötC neurons, on the basis of a small sample in rhythmically active mouse slices, synaptically project to approximately one out of six neurons and form electrical synapses with approximately one out of six neurons that are also type 1 but are different from the first set of neurons $(69,115)$. Each rhythm-generating preBötC neuron also projects an axon toward the midline, presumably to the contralateral preBötC (Figure 3e). Putative rhythm-generating neurons in organotypic cultures of the preBötC form clusters of approximately seven neurons, each projecting to four neighbors. Therefore, connectivity estimates range from $15 \%$ to $67 \%$. The topological pattern of connectivity in neural networks (as in all networks) influences whether and how individual elements, e.g., neurons, can synchronize their activity (116). Therefore, the specific topological pattern of connectivity is at least as important as a simple tally or an estimate of connection probability in understanding network function. For example, the robustness of network function under attack, i.e., destruction of its constituent nodes, depends on topology (117). Cumulative single-neuron laser ablation of $\sim 18 \%$ of inspiratory preBötC neurons causes irreversible cessation of rhythm in slices (118), but to what extent the underlying details of the wiring diagram determine the robustness (fragility) of the rhythmogenic network remains unknown. Robustness is an important characteristic essential both for understanding the neural basis for periodic behaviors like breathing and for interpreting and treating neurodegenerative disorders (119).

\section{The preBötC and the RTN/pFRG Interconnect}

Glutamatergic, Sst-expressing, inspiratory-modulated preBötC neurons project to the RTN/pFRG (27). Glutamatergic, galanin-positive RTN/pFRG neurons project to the preBötC (120). Whether these neurons are involved in the rhythmogenic aspects of the RTN/pFRG remains to be established. The lack of identification of inhibitory connections between these two structures is puzzling; the preBötC and the RTN/pFRG oscillate in antiphase postnatally. A 
polysynaptic inhibitory pathway is possible and may involve a relay in the BötC. Significant resources are being devoted to determining the connectome for cortical columns; the challenges are heightened by the paucity of information concerning the sophisticated signal processing that is occurring. We suggest that the preBötC presents a much more straightforward challenge of considerably less complexity, as the signal processing and output are well understood, and may be useful as an intermediate step in the development of brain connectomics.

\section{GENETICS OF THE RESPIRATORY NETWORK}

Our understanding of the developmental genetics of brain stem circuits underlying the neural control of breathing is rapidly advancing, with considerable potential to inform our understanding of the preBötC and RTN/pFRG. Brain stem organization is remarkably consistent among vertebrates $(121,122)$, a consequence of conserved developmental programs that control stereotyped patterns of gene expression, including critical transcription factors (TFs) (123, 124). At first approximation, every brain stem neuron results from a specific sequence and combination of TF expression on the basis of its physical location during neurogenesis (125). Rostrocaudally, the brain stem is organized into 7 to 8 domains (rhombomeres) associated with conserved and discrete patterns of TF expression (126). Medullary and caudal pontine respiratory populations are distributed across rhombomeres 5-7. Dorsoventrally, the brain stem neural tube can be divided into at least 13 distinct dorsoventral domains on the basis of TF expression (Figure 5a) (127). Each of these domains produces distinct classes of neurons with their own neurotransmitter identity (Figure 5a), morphology, and migratory pattern (Figure 5 b ). Neurons of the ventral respiratory column (119) and the adjacent RTN/pFRG are derived predominantly from four distinct progenitor domains (Figure $5 \boldsymbol{a}-\boldsymbol{c}$ ).

\section{Genetics of the preBötC}

We make the case that the preBötC is the rhythmogenic source of inspiratory drive. Yet it is a small portion of the rostral ventral respiratory column (119), which mostly contains bulbospinal premotoneurons receiving inspiratory drive from the preBötC. What is the developmental mechanism underlying this ventral respiratory column organization, and in particular, what makes the preBötC special? Are its constituent neurons genetically distinct from adjacent respiratory premotoneurons? The expression in preBötC neurons of the receptor NK1R $(91,128-130)$, the peptide Sst $(22,24)$, and the glycoprotein reelin (131) distinguishes this structure from surrounding regions (Figure 5d); yet none of these markers appear to endow the preBötC with rhythmogenic properties. In mice, these genes are expressed on only a partially overlapping minority of preBötC excitatory neurons ( $\sim 1,000$ per side) (132). The role that different genetically defined subclasses of preBötC neurons play in rhythmogenesis is unknown. Genetic ablation of Sst or NK1R genes, but not of the neurons that would otherwise express these genes, does not appear to affect the formation of the preBötC and produces only mildly dysfunctional respiratory phenotypes (133136). No intrinsic membrane properties, e.g., $I_{\mathrm{NaP}}$ or $I_{\mathrm{CAN}}$, appear uniquely expressed in subsets of preBötC neurons.

The ventrolateral medulla (VLM) contains both excitatory (glutamatergic) and inhibitory, mostly glycinergic, respiratory neurons (Figure $5 \boldsymbol{e}, \boldsymbol{f})(59,60,137,138)$. Glutamate is the essential excitatory neurotransmitter for preBötC rhythmogenesis $(31,139)$. Genetic ablation of the vesicular glutamate transporter 2 (VGlut2, Slc17a6) completely eliminates respiratory-related motor activity both in vitro and in vivo (32). Respiratory dysfunction leading to death at or near birth is a consequence of the genetic ablation of a number of TFs $(123,140)$. In some cases (see 
below), these TFs play a role in the specification of neurons that modulate preBötC function, but not in the specification of preBötC neurons. These TFs include those important for the specification of ventral medullary and pontine glycinergic neurons (Lbx1, Ptfla) or glutamatergic neurons (Atoh1, Krox20/Egr2, Lbx1, Lmx1b, MafB/Kreisler, Phox2b, Tlx3, Tshz3) (100, 127, 140-151). None of these mutations, however, affect the specification of preBötC NK1R/Sst neurons. For example, Lmx1b and Tlx3 are expressed in and essential for the formation of ventral medullary catecholaminergic neurons that partially intermingle with preBötC neurons (152). Tlx3 mutation leads in vitro to respiratory instability that is alleviated by disinhibition. Similarly, subsets of Atoh1-derived neurons are present near the ventral medullary surface (153). Atoh1 mutant mice do not breathe in vivo at birth, yet a functional preBötC and rhythmic activity persist in vitro (141).

The TF Dbx1 is essential for preBötC development and respiratory function. Dbx1 is expressed throughout the brain stem and spinal cord in V0 neural progenitor cells (Figure 5a). The V0 domain consists of at least two subdivisions, dorsal and ventral (V0d and V0v) $(154,155)$. V0d neurons are predominantly GABAergic and are located near the dorsal midline. V0v postmitotic neurons express the TFs Evx1 and Evx2, and a subset also express VGlut2. In the spinal cord, V0v neurons are a small population that migrates toward the ventral midline. Within the medulla, however, the V0v glutamatergic subpopulation greatly expands and migrates laterally to the ventral medullary

\section{Figure 5}

Genetic organization of brain stem respiratory regions. $(a)(L e f t)$ Schematized description of brain stem progenitor domains for eight dorsal (dA1-dB4) and five ventral (v0-v3l) progenitor populations based on their relative locations within the brain stem progenitor region. (Middle) A partial list of transcription factors (TFs) expressed at some point within progenitors (in italic font) or postmitotic neurons (in roman font) within each domain. (Right) The neurotransmitter identity of neurons derived from each domain. Adapted from Reference 123. (b) The partial migratory path of ventral medulla (left) and caudal pons (right) neurons in the embryonic mouse brain stem. Colors correspond to those of the domains in panel $a$. Thick arrows correspond to populations important for breathing. The $\mathrm{dB} 2$ population (light green) is present only in the caudal pons. $(c)$ The developmental origin and approximate anatomical locations of respiratory-related populations in the sagittal plane within the ventral medulla and caudal pons. Colors correspond to those of the domains in panel $a$. The legend describes transmitters released by these neurons. Within the ventral respiratory column, nearly all respiratory-related glutamatergic neurons are Dbx1 derived. The dotted red box shows the location of preBötC Sst-expressing neurons. The RTN/pFRG, in contrast, contains Dbx1, Atoh1, and Phox2b glutamatergic populations. The ventral medulla also contains many $\mathrm{dB} 1$-derived glycinergic neurons. $(d)$ preBötC neurons are derived from Dbx1-expressing progenitors. The four-color confocal image shows coexpression of NK1R (magenta), Sst2aR (green), Sst (cyan), and $\beta$-gal (yellow) in the P0 Dbx1 $\beta$-gal mouse (adapted from Reference 132). Arrows indicate coexpression of all four genes. The red arrowhead indicates a Dbx1-derived, NK1R/Sst2aR-expressing neuron that lacks Sst. The yellow arrowheads indicate Dbx1-derived neurons lacking coexpression. The magenta arrowhead indicates a NK1R-expressing nucleus ambiguus neuron. (e) preBötC Dbx1 neurons are glutamatergic. The image shows $\beta$-gal (magenta) expression within the majority of VGlut2 (green)-expressing preBötC neurons (adapted from Reference 132). The inset is enlarged from the square in the main image. Arrows indicates coexpression. Arrowheads indicate absence of coexpression. $(f)$ preBötC contains glycinergic neurons. The three-color confocal image shows Pax2 (red) and Sst (blue) immunoreactivity with intrinsic GFP from a P0 GlyT2-GFP transgenic mouse (59). Pax2 (red arrowheads) colocalizes with Sst (magenta arrowheads) or GFP (yellow arrowheads), but there is no GFP expression in Sst neurons. The inset is enlarged from the square in the main image. Scale bars in panels $c, d, e$, and $f$ : $200 \mu \mathrm{m}$. D denotes dorsal, and L denotes lateral. $(\mathrm{g})$ RTN/pFRG Phox2b neurons express Phox 2b and Atoh1. (i) Magnification of the

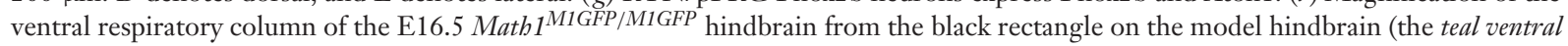
region is the pFRG/RTN, whereas the yellow circle indicates the preBötC), showing NK1R (red), Phox2b (blue), and Math1-EGFP ( green) expression. NK1R is expressed in both pFRG/RTN and preBötC neurons. Magnified pFRG/RTN neurons from the caudal pole of VII (white rectangle in panel $i$ ) show colocalization of Math1-EGFP with Phox2b and NK1R. (ii) The three markers are merged. Further magnification from the white box in panel $i i$ is shown in panel $i i^{\prime}$. Image from Reference 141 . Abbreviations: $\beta$-gal, $\beta$-galactosidase, A5, A5 catecholaminergic group; ACh, acetylcholine; BötC, Bötzinger Complex; cb, cerebellum; GABA, gamma aminobutyric acid; GFP, green fluorescent protein; Glu, glutamate; Gly, glycine; LRN, lateral reticular nucleus; me, medulla; NA, nucleus ambiguus; NK1R, neurokinin 1 receptor; pn, pons; preBötC, preBötzinger Complex; RTN/pFRG, retrotrapezoid nucleus/parafacial respiratory group; rVRG, rostral ventral respiratory group; Sst, somatostatin; Sst2aR, Sst2a receptor; VIIn, facial motonucleus; VGlut2, vesicular glutamate transporter 2. 
a Domain

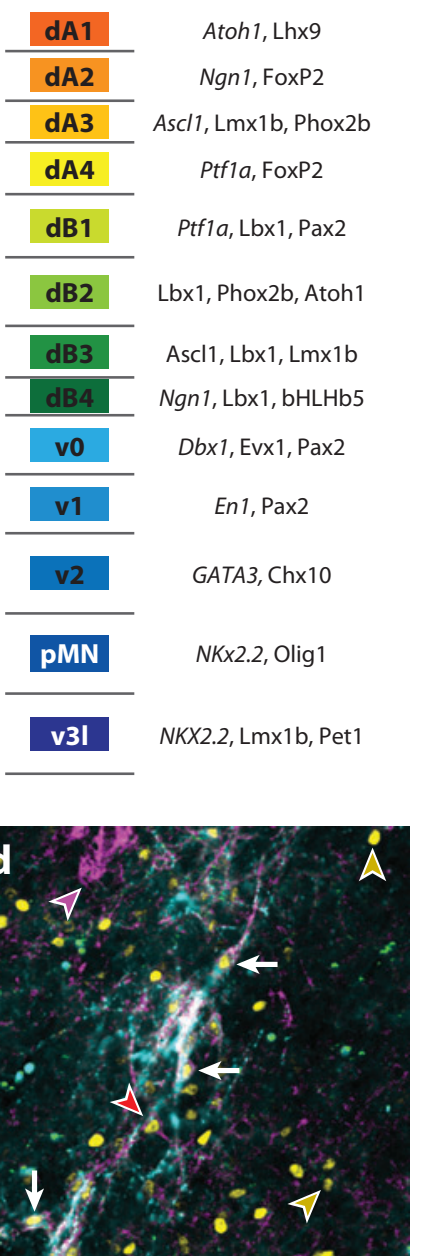

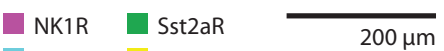

Transmitter

Glu

Glu

Glu, NA

Glu

GABA, Gly

Glu

Glu

GABA

Glu, GABA

GABA, Gly

Glu, GABA

$\mathrm{ACh}$

$5-\mathrm{HT}$ b E12.5 mouse

Ventral medulla

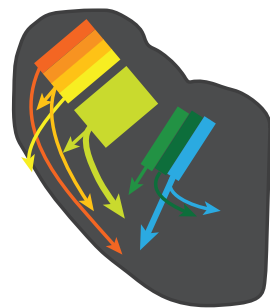

Caudal pons

C P0 mouse

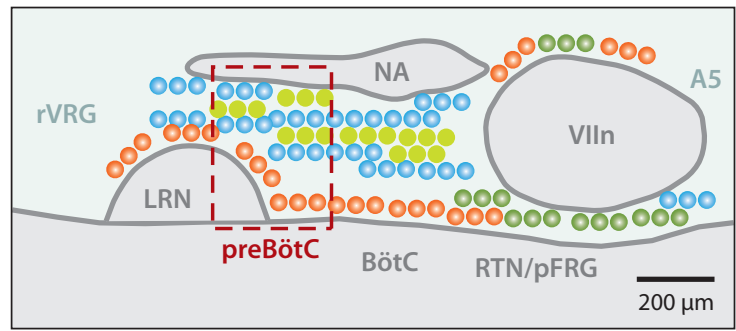

OdA1Glu $\bigcirc$ voGlu $\quad$ dB1 Gly $\bigcirc$ dB2 Glu

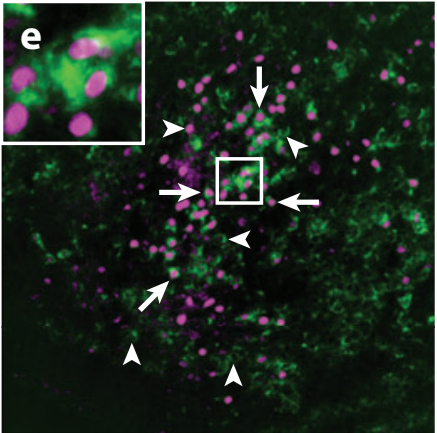

Dbx1 $\beta$-gal VGlut2 $\overline{200 \mu \mathrm{m}}$

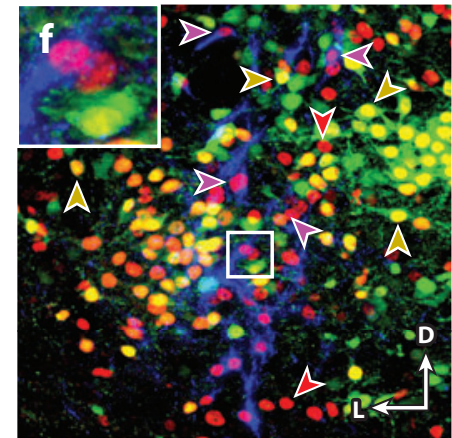

GlyT2-GFP Pax2 Sst

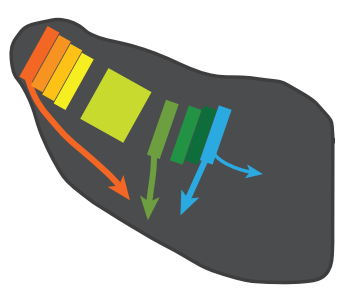

Sst Dbx1 $\beta$-gal

Math1 M1GFP/M1GFP (E16.5)

g
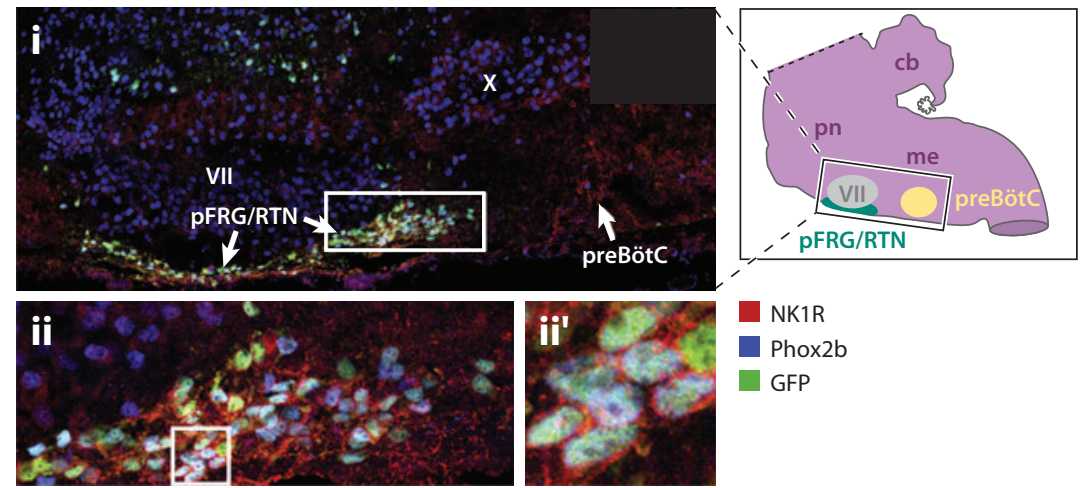
surface, producing a column extending the full length of the ventral respiratory column and including the RTN/pFRG, BötC, preBötC, and rostral ventral respiratory group (Figure $\mathbf{5} \boldsymbol{b}-\boldsymbol{e}$ ).

Neurons with high levels of Sst in the neonatal mouse ventral medulla are limited to the (approximate boundaries of the) preBötC and are derived from Dbx1-expressing progenitors (Figure $5 \boldsymbol{d})(132,156)$. These cells are $\sim 20 \%$ of preBötC Dbx1-derived neurons. The function and developmental relationship between preBötC Sst and other neurons within and adjacent to the preBötC are unknown. NK1Rs and somatostatin 2a receptors (Sst2aRs) are coexpressed on the majority of preBötC Sst neurons but are more broadly expressed within and adjacent to the preBötC (Figure $5 \boldsymbol{d}$ ). In addition, Dbx1 defines not only preBötC NK1R/Sst-expressing neurons but essentially all glutamatergic neurons of the ventral respiratory column (Figure 5e). This finding suggests that, for breathing, rhythm-generating neurons and (a significant proportion of the) downstream premotor neurons are derived from a single developmental progenitor domain. This organization is in contrast to that proposed for models of locomotion in which rhythms are generated by the interactions of multiple developmental populations $(86,157)$. The genetic mechanisms specifying preBötC neurons from the larger Dbx1-derived population are unknown, but they may induce signaling cascades similar to those responsible for segmental patterning of homeobox genes in the brain stem and spinal cord (158).

The elimination of the commissural projections from only Dbx1-derived neurons by deletion of the axon guidance receptor Robo desynchronizes respiratory outflow between the left and right sides (Figure 3e) (156). Genetic deletion of Dbx1 eliminates all preBötC glutamatergic respiratory neurons, including those expressing NK1R and Sst, with consequent complete elimination of inspiratory activity, both in vitro and in vivo $(132,156)$. Additionally, preBötC Dbx1-derived neurons are predominantly inspiratory modulated, at least in vitro (Figure $3 \boldsymbol{d})(132,156)$. Together, these data indicate that both the generation and the coordination of inspiratory output depend on commissurally projecting Dbx1-derived neurons that are presumably within the preBötC (Figure $3 \boldsymbol{e}$ ).

\section{The Mouse in the Room}

In vivo experiments targeting respiratory neurons in goats suggest that under certain conditions, breathing does not require preBötC neurons (159-162). Here, a nonspecific excitotoxin, ibotenic acid (IA), is injected in slowly increasing quantities over 5 weeks (to avoid bilateral application of high dosages that can cause acute cardiac and respiratory failure). IA affects breathing during injection, but breathing returns to normal by the next day. This protocol results in a substantial, if not complete, loss of neurons within the presumptive preBötC, at least to the degree that markers and neuroanatomy in goats are homologous to those in rodents. In contrast, goats injected with SP-saporin into the same region followed 10-14 days later with a single large dose of IA cease breathing for at least $6 \mathrm{~h}$ without recovery, either leading to death or necessitating euthanasia. One interpretation is that normal breathing can be generated in the absence of preBötC neurons, given modest damage per event and sufficient time for respiratory network reorganization between events that possibly include neurogenesis. There are four alternative explanations. $(a)$ With sufficient time, regions outside of the preBötC with a different developmental lineage can generate normal respiratory output. One possible source is neurons in the RTN/pFRG (see below). (b) The postlesion rhythm may be generated by neurons that are genetically related to preBötC neurons but that, at least in rats, do not normally generate inspiratory rhythm (24), i.e., some subset of the Dbx1-derived glutamatergic neurons that extend the length of the ventral respiratory column (Figure 5b). This last explanation is consistent with the observation that saporin ablations that include but extend beyond the preBötC lead more rapidly to death than do smaller ablations (20, 163). (c) In goats, the boundaries of the preBötC, which have been well characterized only in 
the rat and the mouse, extend beyond the IA lesion, and a prolonged recovery is required for the unlesioned remaining neurons to coordinate their activity for effective breathing movements. NK1R expression is not limited to the preBötC in mice, and in goats NK1R expression appears to be ubiquitous, so the use of NK1R expression to identify preBötC anatomical boundaries is questionable (162). (d) The rhythm is generated by a population at some distance from the preBötC, perhaps in the rostral pons, although there are currently no data to support this.

\section{GENETICS OF THE RTN/pFRG: ACTIVE EXPIRATION, CENTRAL CHEMORECEPTION, AND CONGENITAL CENTRAL HYPOVENTILATION SYNDROME}

There are two critical functions associated with the RTN/pFRG: the generation of active expiration and central chemoreception. The lateral RTN/pFRG is the most sensitive site for the induction of active expiration without a concomitant increase in inspiration (106); this region contains few, if any, Phox $2 \mathrm{~b}$ neurons (Figure 5a,c). One of the most essential functions of breathing in mammals is maintenance of the partial pressure of blood/tissue $\mathrm{CO}_{2}$ (at $\sim 40 \mathrm{~mm} \mathrm{Hg}$ in arterial blood in humans). Several brain stem populations of neurons, in particular the medial RTN/pFRG and the raphe, likely play a role in this central chemosensitivity (15). Blunted $\mathrm{CO}_{2}$ chemosensitivity in humans is a defining characteristic of congenital central hypoventilation syndrome (CCHS) (164). CCHS is a rare disorder caused by an alanine expansion in the gene encoding Phox $2 \mathrm{~b}$, a highly conserved and essential TF involved in the specification of brain stem visceral sensory and motoneurons (148). The size of the Phox $2 \mathrm{~b}$ expansion directly correlates with the severity of CCHS. In rodents, a small population of $\sim 2,100$ RTN/pFRG glutamatergic neurons are responsive to small changes in $\mathrm{pH}$ and express Phox $2 \mathrm{~b}(99,100)$. This identification of a specific population of neurons as putative $\mathrm{CO}_{2}$ sensors is a landmark finding of the first developmental marker for neurons that are important for breathing and whose dysfunction leads to a survivable breathing phenotype (147).

The case that RTN/pFRG neurons play a role in mediating chemosensitivity is compelling. Briefly, the elimination of $\sim 70 \%$ of RTN/pFRG Phox $2 \mathrm{~b}$ neurons, by the use of the aforementioned saporin lesion technique, leads to an $\sim 50 \%$ attenuation of the ventilatory response to increases in blood $\mathrm{pH}$ (165). Activation of medial RTN/pFRG Phox $2 \mathrm{~b}$ neurons directly increases inspiratory output (166) and can produce active expiration (167). In contrast, stimulation of all neurons in the non-Phox $2 \mathrm{~b}$-expressing lateral RTN/pFRG reliably evokes active expiration but has little effect on inspiratory activity (106). In the adult rat, medial RTN/pFRG Phox2b neurons are not rhythmically active, even under conditions of strong chemosensitive drive. This is consistent with a role of the medial RTN/pFRG in chemosensitivity but not in rhythmogenesis or the production of active expiration $(168,169)$.

Like nearly all TFs, Phox $2 \mathrm{~b}$ is expressed in distinct subpopulations, only some of which maintain postnatal expression $(170,171)$. RTN/pFRG Phox $2 \mathrm{~b}$ neurons are generated from a caudal pontine progenitor population that coexpresses the TF Lbx1 $(127,143)$. These neurons migrate to their final location in the VLM (dB2 in Figure $5 \boldsymbol{b}, \boldsymbol{f}$ ). The RTN/pFRG is absent in Lbx1 mutant mice as well as in Krox20-null mice that lack rhombomeres 3 and 5 (127, 143, 172). Phox2b gene function is necessary for RTN/pFRG formation, as its conditional elimination by using either Lbx1 (limited to the dB2 domain; see Figure 5a) or Krox20 (limited to rhombomeres 3 and 5) cre recombinase mice prevents RTN formation and produces neonatal lethality and/or blunted chemosensitivity $(147,173)$.

Mouse mutants that recapitulate the most common CCHS mutation, an alanine expansion $\left(\mathrm{Phox} 2 \mathrm{~b}^{27 \mathrm{Ala}}\right)$ in the germline, have a blunted inspiratory response to elevated $\mathrm{CO}_{2}$ in in vitro 
embryonic preparations. Although such mice are capable of at least some inspiratory activity, most die at or shortly after birth $(147,173)$. In this mutation, RTN/pFRG Phox2B neurons form dorsally but do not migrate to the ventral surface. This migration normally coincides with the expression of the TF Atoh1 within putative RTN/pFRG neurons, but Atoh1 expression is lost in these mice $(141,153,173,174)$. Loss of Atoh1 also prevents RTN/pFRG migration (141, 153, 173 , 174). Surprisingly, the conditional expression of the Phox $2 \mathrm{~b}^{27 \mathrm{Ala}}$ mutation within rhombomeres 3 and 5 is not neonatal lethal but results in the loss of Atoh1 expression and of RTN/pFRG neuron migration, as well as in blunted chemosensitivity in neonates. By 4 months of age, however, mice recover $60 \%$ of their $\mathrm{CO}_{2}$ sensitivity (compared with wild-type controls) and have normal blood $\mathrm{CO}_{2}$ levels at rest (173).

\section{FETAL DEVELOPMENT OF THE RTN/PFRG}

In utero, rhythmic respiratory motor output in mice begins at approximately embryonic day 15.5 (E15.5) coincident with the onset of respiratory activity in the embryonic preBötC (175). However, rhythmic brain stem activity is present by E14.5, i.e., $\sim 1$ day prior to the onset of preBötC activity, within the Phox $2 \mathrm{~b}$-expressing region near the facial nucleus. This region is termed the embryonic parafacial oscillator (ePF) and is presumably the embryonic equivalent of the RTN/pFRG. In the later fetal period, the initially slow preBötC rhythm is coupled to a faster ePF rhythm (145). Unlike the preBötC rhythm, ePF rhythms do not require glutamatergic neurotransmission, as they persist following pharmacological blockade of glutamate receptors or genetic ablation of the glutamate transporter VGlut2 $(32,145)$. Rhythmically active ePF neurons express Phox2b and NK1R, but not Sst2a or $\mu$-opioid receptors (132). In neonatal en bloc preparations, the majority of pFRG pre-I and respiratory-modulated tonic neurons express Phox2b (176, 177). Mutations that eliminate RTN/pFRG Phox2b neurons, such as those in Krox20 mutants, eliminate ePF activity, resulting in a slowed fetal preBötC rhythm (145). Together these data suggest that ePF and RTN/pFRG contain the same population of Phox2b-expressing neurons that have a vital perinatal role. As the ePF is an intrinsically rhythmic population coupled to breathing movements, its neurons represent excellent candidates for an evolutionarily conserved, independent expiratory oscillator (178). Significantly, however, fetal RTN/pFRG rhythms persist in Dbx1 mutant mice. These mice do not generate any rhythmic activity from VII or other respiratory motoneurons, suggesting that RTN/pFRG neurons alone are insufficient to assure the generation of active expiratory (or inspiratory) movements (156).

\section{SEROTONIN, CENTRAL CHEMORECEPTION, AND SUDDEN INFANT DEATH SYNDROME}

Raphe serotonergic neurons are also proposed to play a role in mediating $\mathrm{CO}_{2}$ chemosensitivity (179). Raphe neurons have a discrete pattern of TF expression, including the combinatorial expression of the TFs Pet1 and Lmx1b (v3l in Figure 5a). Pet1 is expressed only in serotonin neurons, and its genetic ablation decreases their number by $70 \%$ (180). Lmx1b is expressed in several brain stem populations, but its conditional elimination from Pet1-expressing neurons completely eliminates all brain stem serotonergic neurons. In adult mice, such elimination produces an $\sim 50 \%$ reduction in the ventilatory response to high levels of $\mathrm{CO}_{2}$ but has no effect on baseline respiration (181). This is the same pattern of chemosensitivity seen in adult mice with Phox $2 b^{27 A l a /+}$ RTN/pFRG mutations. Partial serotonin neuron loss produces a similar but smaller effect (182). In neonates, the complete loss of serotonin increases and prolongs neonatal apnea and reduces ventilation at rest; ventilation nevertheless recovers to wild-type levels by adulthood (183). Selective silencing of 
raphe neurons in adults depresses breathing and blunts chemosensitivity, indicating a continuing role for these neurons in respiratory modulation (184). In aggregate, these data strongly suggest that both RTN/pFRG and raphe neurons contribute to normal chemosensitivity and that the explicit role of either one may depend upon the age and state (e.g., sleep-wake, rest-exercise) of the animal (185). Medullary serotonergic neurons are present in vitro and can affect rhythmogenesis and motor output $(28,186,187)$, representing mechanisms by which serotonin can regulate $\mathrm{CO}_{2}$.

Serotonin dysfunction is hypothesized to underlie many cases of sudden infant death syndrome (SIDS) (188). In vitro, serotonin induces a depolarizing, nonselective cation current in preBötC neurons that enhances burst generation (65). The same or a very similar nonselective cation current is also a target of SP and is likely coreleased (under unknown conditions) by raphe neurons (40, $44,115)$. These serotonergic inputs also enhance bursting pacemaker neurons by amplifying $I_{\mathrm{NaP}}$ and closing leakage $\mathrm{K}^{+}$currents $(65,189)$. Although the molecular identity of the nonselective cation current(s) described in these various studies is unknown, the current may be related to the leakage $\mathrm{Na}^{+}$current (190) or to a canonical transient receptor potential channel (TRPC) such as TRPC3 or TRPC7 (40).

\section{ASTROCYTES AND CHEMOSENSATION}

The mechanism(s) underlying central chemosensitivity in vivo is incompletely understood. In highly reduced preparations, both raphe and RTN/pFRG neurons are chemosensitive, but no protein or pathway within either of these populations has been unequivocally identified as the transducer of $\mathrm{pH} / \mathrm{CO}_{2}$ sensitivity. Hence whether these neurons are intrinsic chemosensors or part of a relay pathway is unclear, although the two mechanisms are not mutually exclusive and appear to act in concert through multiple transduction mechanisms (185). An alternate hypothesis is that $\mathrm{pH}$ and $\mathrm{CO}_{2}$ are intrinsically sensed by a distinct population of astrocytes, which, by releasing ATP near the ventral surface, modulate the activity of nearby chemosensitive neurons and thus provide a $\mathrm{CO}_{2}$-related stimulus that modulates breathing (191). The ventral medullary surface has several sites where local acidification increases ventilation $(192,193)$. At some of these sites, including adjacent to the RTN/pFRG, astrocytes respond to modest acidification $(\sim 0.2 \mathrm{pH}$ units) by releasing ATP. Also, there is an increase in ATP release as blood $\mathrm{CO}_{2}$ increases in vivo, and local application of ATP receptor agonists increases ventilation (194). Optogenetic stimulation of astrocytes in the RTN/pFRG increases ventilation as well (191). A subset of brain stem astrocytes, including those adjacent to the RTN/pFRG, express connexin 26 (Cx26). Cx26 hemichannels are directly $\mathrm{CO}_{2}$ sensitive and open to release ATP upon acidification $(195,196)$, and blocking gap junctions reduces $\mathrm{CO}_{2}$ sensitivity akin to the action of purinergic receptor antagonists (197). Astrocytic gliotransmission thus provides a hypothetical mechanism linking $\mathrm{CO}_{2}$ levels to changes in breathing.

\section{RESPIRATORY NETWORKS AND HUMAN DISEASE}

Increasingly, researchers working on the neural control of breathing are trying to understand the causes of, and to find rational therapies for, diseases that affect breathing. Central respiratory dysfunction is symptomatic of many diseases that can occur at almost any time during life, and unfortunately, we poorly understand the underlying causes $(198,199)$. This is true even for diseases in which causative single-gene mutations are known and relevant transgenic mice are available; examples include CCHS (see above), Rett syndrome, and Joubert syndrome (200-202). In Rett syndrome, due to a mutation in the MeCP2 gene, girls and, much more rarely, boys show prolonged periods of respiratory dysfunction during wakefulness and milder effects during sleep 
$(203,204)$. MeCP2 mutant mice show similar respiratory deficits, and dysfunction in several different populations of glutamatergic and noradrenergic neurons is suggested as causative, although the respiratory phenotype may not manifest by effects in the brain stem or glia (203, 205-208). Similarly, breathing pathologies, especially prolonged and frequent apneas during sleep, are present in a number of neurodegenerative diseases such as Parkinson's, amyotrophic lateral sclerosis, and multiple-systems atrophy (MSA). Losses of putative preBötC NK1R or Sst neurons have been found in both Parkinson's- and MSA-affected brains (119, 209-212).

More problematic are diseases that likely have a strong, but currently unknown, genetic component(s), such as ROHHAD (rapid-onset obesity, hypoventilation, and hypothalamic dysfunction), and diseases in which the role of genetics is unknown, such as apnea of prematurity, Perry syndrome, SIDS, and sleep apnea (198, 213-215, 216, 217). These last two are the most prevalent diseases with a breathing phenotype: For live births, SIDS kills $\sim 6$ in 1,000 infants $<1$ year of age (http://www.sidscenter.org/Statistics/table1.html), and sleep apnea affects 4-6\% of the adult male population at all ages and an equivalent percentage of females postmenopause (http://www.rightdiagnosis.com/s/sleep_disorders/prevalence-types.htm). Sleep apnea is associated with significant increases in morbidity and mortality. As mentioned above, a leading hypothesis posits that SIDS is a dysfunction of serotonin in the infant brain stem (216). The relatively early stage of our knowledge of the respiratory neural control system as it relates to human disease has led to confusion about the underlying causes, which may consequently overemphasize possible future treatments to the detriment of both scientific progress and patient hopes.

\section{CHALLENGES REMAINING}

Although great progress has been made since the discovery of the preBötC, especially as related to key and specific brain stem sites generating and modulating respiratory rhythm and pattern, our understanding of the underlying mechanisms is still at an early stage. Key high-priority problems include identifying and understanding neurons important for the generation of expiratory activity and understanding the mechanisms of rhythm generation within the preBötC. Of all vital behaviors in mammals, the neural control of breathing may be the first to be solved.

\section{DISCLOSURE STATEMENT}

The authors are not aware of any affiliations, memberships, funding, or financial holdings that might be perceived as affecting the objectivity of this review.

\section{ACKNOWLEDGMENTS}

We apologize to the many colleagues whose original work could not be cited because of length restrictions. This work was supported by research and training grants from the NINDS and NHLBI (NIH), the NSF, and the Parker B. Francis Foundation.

\section{LITERATURE CITED}

1. Pattinson KT, Mitsis GD, Harvey AK, Jbabdi S, Dirckx S, et al. 2009. Determination of the human brainstem respiratory control network and its cortical connections in vivo using functional and structural imaging. Neuroimage 44:295-305

2. Ruangkittisakul A, Panaitescu B, Ballanyi K. 2011. $\mathrm{K}^{+}$and $\mathrm{Ca}^{2+}$ dependence of inspiratory-related rhythm in novel "calibrated" mouse brainstem slices. Respir. Physiol. Neurobiol. 175:37-48 
3. Ruangkittisakul A, Schwarzacher SW, Secchia L, Ma Y, Bobocea N, et al. 2008. Generation of eupnea and sighs by a spatiochemically organized inspiratory network. 7. Neurosci. 28:2447-58

4. Ruangkittisakul A, Secchia L, Bornes TD, Palathinkal DM, Ballanyi K. 2007. Dependence on extracellular $\mathrm{Ca}^{2+} / \mathrm{K}^{+}$antagonism of inspiratory centre rhythms in slices and en bloc preparations of newborn rat brainstem. 7. Physiol. 584:489-508

5. Smith JC, Ellenberger HH, Ballanyi K, Richter DW, Feldman JL. 1991. Pre-Bötzinger Complex: a brainstem region that may generate respiratory rhythm in mammals. Science 254:726-29

6. Del Negro CA, Kam K, Hayes JA, Feldman JL. 2009. Asymmetric control of inspiratory and expiratory phases by excitability in the respiratory network of neonatal mice in vitro. 7. Physiol. 587:1217-31

7. Rekling JC, Champagnat J, Denavit-Saubie M. 1996. Electroresponsive properties and membrane potential trajectories of three types of inspiratory neurons in the newborn mouse brain stem in vitro. 7 . Neurophysiol. 75:795-810

8. Feldman JL. 1986. Neurophysiology of breathing in mammals. In Handbook of Physiology, Section I: The Nervous System, Volume IV: Intrinsic Regulatory Systems of the Brain, ed. FE Bloom, pp. 463-524. Bethesda, MD: Am. Physiol. Soc.

9. Paton JF. 1996. A working heart-brainstem preparation of the mouse. F. Neurosci. Methods 65:63-68

10. Lieske SP, Thoby-Brisson M, Telgkamp P, Ramirez JM. 2000. Reconfiguration of the neural network controlling multiple breathing patterns: eupnea, sighs and gasps. Nat. Neurosci. 3:600-7

11. Paton JF, Abdala AP, Koizumi H, Smith JC, St-John WM. 2006. Respiratory rhythm generation during gasping depends on persistent sodium current. Nat. Neurosci. 9:311-13

12. Viemari JC, Garcia AJ 3rd, Doi A, Ramirez JM. 2011. Activation of alpha-2 noradrenergic receptors is critical for the generation of fictive eupnea and fictive gasping inspiratory activities in mammals in vitro. Eur. 7. Neurosi. 33:2228-37

13. Suzue T. 1984. Respiratory rhythm generation in the in vitro brain stem-spinal cord preparation of the neonatal rat. 7. Physiol. 354:173-83

14. Feldman JL, Connelly CA, Ellenberger HH, Smith JC. 1990. The cardiorespiratory system within the brainstem. Eur. F. Neurosci. Suppl. 3:171

15. Feldman JL, Mitchell GS, Nattie EE. 2003. Breathing: rhythmicity, plasticity, chemosensitivity. Annu. Rev. Neurosci. 26:239-66

16. Rekling JC, Feldman JL. 1998. PreBötzinger Complex and pacemaker neurons: hypothesized site and kernel for respiratory rhythm generation. Annu. Rev. Physiol. 60:385-405

17. Feldman JL, Del Negro CA. 2006. Looking for inspiration: new perspectives on respiratory rhythm. Nat. Rev. Neurosci. 7:232-42

18. Janczewski WA, Feldman JL. 2006. Distinct rhythm generators for inspiration and expiration in the juvenile rat. F. Physiol. 570:407-20

19. Smith JC, Abdala AP, Koizumi H, Rybak IA, Paton JF. 2007. Spatial and functional architecture of the mammalian brain stem respiratory network: a hierarchy of three oscillatory mechanisms. 7 . Neurophysiol. 98:3370-87

20. Gray PA, Janczewski WA, Mellen N, McCrimmon DR, Feldman JL. 2001. Normal breathing requires preBötzinger Complex neurokinin-1 receptor-expressing neurons. Nat. Neurosci. 4:927-30

21. McKay LC, Janczewski WA, Feldman JL. 2005. Sleep-disordered breathing after targeted ablation of preBötzinger Complex neurons. Nat. Neurosci. 8:1142-44

22. Stornetta RL, Rosin DL, Wang H, Sevigny CP, Weston MC, Guyenet PG. 2003. A group of glutamatergic interneurons expressing high levels of both neurokinin-1 receptors and somatostatin identifies the region of the pre-Bötzinger Complex. F. Comp. Neurol. 455:499-512

23. Llona I, Ampuero E, Eugenin JL. 2004. Somatostatin inhibition of fictive respiration is modulated by pH. Brain Res. 1026:136-42

24. Tan W, Janczewski WA, Yang P, Shao XM, Callaway EM, Feldman JL. 2008. Silencing preBötzinger Complex somatostatin-expressing neurons induces persistent apnea in awake rat. Nat. Neurosci. 11:53840

25. Grillner S, Jessell TM. 2009. Measured motion: searching for simplicity in spinal locomotor networks. Curr. Opin. Neurobiol. 19:572-86 
26. Gossard JP, Dubuc R, Kolta A. 2011. Preface. Breathe, walk and chew: the neural challenge: part II. Prog. Brain Res. 188:ix

27. Tan W, Pagliardini S, Yang P, Janczewski WA, Feldman JL. 2010. Projections of preBötzinger Complex neurons in adult rats. F. Comp. Neurol. 518:1862-78

28. Bianchi AL, Denavit-Saubie M, Champagnat J. 1995. Central control of breathing in mammals: neuronal circuitry, membrane properties, and neurotransmitters. Physiol. Rev. 75:1-45

29. von Euler C. 1986. Brain stem mechanisms for generation and control of breathing pattern. In Handbook of Physiology, Section 3: The Respiratory System, Volume II: Control of Breathing, ed. A Fishman, N Cherniak, J Widdicombe, pp. 1-67. Bethesda: Am. Physiol. Soc.

30. Richter DW. 1982. Generation and maintenance of the respiratory rhythm. F. Exp. Biol. 100:93-107

31. Funk GD, Smith JC, Feldman JL. 1993. Generation and transmission of respiratory oscillations in medullary slices: role of excitatory amino acids. F. Neurophysiol. 70:1497-515

32. Wallen-Mackenzie A, Gezelius H, Thoby-Brisson M, Nygard A, Enjin A, et al. 2006. Vesicular glutamate transporter 2 is required for central respiratory rhythm generation but not for locomotor central pattern generation. 7. Neurosci. 26:12294-307

33. Gossard JP, Dubuc R, Kolta A, eds. 2010. Breathe, Walk and Chew: The Neural Challenge: Part I (Prog. Brain Res. 187:2-230). New York: Elsevier

34. Tazerart S, Vinay L, Brocard F. 2008. The persistent sodium current generates pacemaker activities in the central pattern generator for locomotion and regulates the locomotor rhythm. $\mathcal{F}$. Neurosci. 28:8577-89

35. Brocard F, Verdier D, Arsenault I, Lund JP, Kolta A. 2006. Emergence of intrinsic bursting in trigeminal sensory neurons parallels the acquisition of mastication in weanling rats. F. Neurophysiol. 96:2410-24

36. Best J, Borisyuk A, Rubin J, Terman D, Wechselberger M. 2005. The dynamic range of bursting in a model respiratory pacemaker network. SIAM J. App. Dyn. Sys. 4:1107-39

37. Purvis LK, Smith JC, Koizumi H, Butera RJ. 2007. Intrinsic bursters increase the robustness of rhythm generation in an excitatory network. F. Neurophysiol. 97:1515-26

38. Koizumi H, Smith JC. 2008. Persistent $\mathrm{Na}^{+}$and $\mathrm{K}^{+}$-dominated leak currents contribute to respiratory rhythm generation in the pre-Bötzinger Complex in vitro. F. Neurosci. 28:1773-85

39. Ramirez JM, Koch H, Garcia AJ 3rd, Doi A, Zanella S. 2011. The role of spiking and bursting pacemakers in the neuronal control of breathing. 7. Biol. Phys. 37:241-61

40. Ben-Mabrouk F, Tryba AK. 2010. Substance P modulation of TRPC3/7 channels improves respiratory rhythm regularity and $\mathrm{I}_{\mathrm{CAN}}$-dependent pacemaker activity. Eur. F. Neurosci. 31:1219-32

41. Tryba AK, Peña F, Lieske SP, ViemariJC, Thoby-Brisson M, RamirezJM. 2008. Differential modulation of neural network and pacemaker activity underlying eupnea and sigh-breathing activities. $\mathcal{F}$. Neurophysiol. 99:2114-25

42. Peña F, Parkis MA, Tryba AK, Ramirez JM. 2004. Differential contribution of pacemaker properties to the generation of respiratory rhythms during normoxia and hypoxia. Neuron 43:105-17

43. Wittmeier S, Song G, Duffin J, Poon CS. 2008. Pacemakers handshake synchronization mechanism of mammalian respiratory rhythmogenesis. Proc. Natl. Acad. Sci. USA 105:18000-5

44. Peña F, Ramirez JM. 2004. Substance P-mediated modulation of pacemaker properties in the mammalian respiratory network. F. Neurosci. 24:7549-56

45. Brockhaus J, Ballanyi K. 1998. Synaptic inhibition in the isolated respiratory network of neonatal rats. Eur. 7. Neurosci. 10:3823-39

46. Feldman JL, Smith JC. 1989. Cellular mechanisms underlying modulation of breathing pattern in mammals. Ann. NY Acad. Sci. 563:114-30

47. Ren J, Greer JJ. 2006. Modulation of respiratory rhythmogenesis by chloride-mediated conductances during the perinatal period. 7 . Neurosci. 26:3721-30

48. Shao XM, Feldman JL. 1997. Respiratory rhythm generation and synaptic inhibition of expiratory neurons in pre-Bötzinger Complex: differential roles of glycinergic and GABAergic neural transmission. 7. Neurophysiol. 77:1853-60

49. Del Negro CA, Morgado-Valle C, Hayes JA, Mackay DD, Pace RW, et al. 2005. Sodium and calcium current-mediated pacemaker neurons and respiratory rhythm generation. F. Neurosci. 25:446-53

50. Thoby-Brisson M, Ramirez JM. 2001. Identification of two types of inspiratory pacemaker neurons in the isolated respiratory neural network of mice. 7. Neurophysiol. 86:104-12 
51. Del Negro CA, Koshiya N, Butera RJ Jr, Smith JC. 2002. Persistent sodium current, membrane properties and bursting behavior of pre-Bötzinger Complex inspiratory neurons in vitro. $\mathcal{F}$. Neurophysiol. 88:2242-50

52. Del Negro CA, Hayes JA, Pace RW, Brush BR, Teruyama R, Feldman JL. 2010. Synaptically activated burst-generating conductances underlie a group-pacemaker mechanism for respiratory rhythm generation in mammal. See Ref. 33, pp. 111-36

53. Butera RJ Jr, Rinzel J, Smith JC. 1999. Models of respiratory rhythm generation in the pre-Bötzinger Complex. I. Bursting pacemaker neurons. 7. Neurophysiol. 82:382-97

54. Koizumi H, Wilson CG, Wong S, Yamanishi T, Koshiya N, Smith JC. 2008. Functional imaging, spatial reconstruction, and biophysical analysis of a respiratory motor circuit isolated in vitro. $\mathcal{F}$. Neurosci. 28:2353-65

55. Johnson SM, Smith JC, Funk GD, Feldman JL. 1994. Pacemaker behavior of respiratory neurons in medullary slices from neonatal rat. 7. Neurophysiol. 72:2598-608

56. Ramirez JM, Tryba AK, Peña F. 2004. Pacemaker neurons and neuronal networks: an integrative view. Curr. Opin. Neurobiol. 14:665-74

57. Viemari JC, Ramirez JM. 2006. Norepinephrine differentially modulates different types of respiratory pacemaker and nonpacemaker neurons. 7. Neurophysiol. 95:2070-82

58. Pagliardini S, Adachi T, Ren J, Funk GD, Greer JJ. 2005. Fluorescent tagging of rhythmically active respiratory neurons within the pre-Bötzinger Complex of rat medullary slice preparations. 7 . Neurosci. 25:2591-96

59. Morgado-Valle C, Baca SM, Feldman JL. 2010. Glycinergic pacemaker neurons in preBötzinger Complex of neonatal mouse. 7. Neurosci. 30:3634-39

60. Winter SM, Fresemann J, Schnell C, Oku Y, Hirrlinger J, Hulsmann S. 2009. Glycinergic interneurons are functionally integrated into the inspiratory network of mouse medullary slices. Pflïg. Arch. 458:45969

61. Del Negro CA, Morgado-Valle C, Feldman JL. 2002. Respiratory rhythm: an emergent network property? Neuron 34:821-30

62. Pace RW, Mackay DD, Feldman JL, Del Negro CA. 2007. Role of persistent sodium current in mouse preBötzinger Complex neurons and respiratory rhythm generation. 7. Physiol. 580:485-96

63. St-John WM, Waki H, Dutschmann M, Paton JF. 2007. Maintenance of eupnea of in situ and in vivo rats following riluzole: a blocker of persistent sodium channels. Respir. Physiol. Neurobiol. 155:97-100

64. Ptak K, Zummo GG, Alheid GF, Tkatch T, Surmeier DJ, McCrimmon DR. 2005. Sodium currents in medullary neurons isolated from the pre-Bötzinger Complex region. 7. Neurosci. 25:5159-70

65. Ptak K, Yamanishi T, Aungst J, Milescu LS, Zhang R, et al. 2009. Raphe neurons stimulate respiratory circuit activity by multiple mechanisms via endogenously released serotonin and substance P. F. Neurosci. 29:3720-37

66. Brocard F, Tazerart S, Vinay L. 2010. Do pacemakers drive the central pattern generator for locomotion in mammals? Neuroscientist 16:139-55

67. Rubin JE, Hayes JA, Mendenhall JL, Del Negro CA. 2009. Calcium-activated nonspecific cation current and synaptic depression promote network-dependent burst oscillations. Proc. Natl. Acad. Sci. USA 106:2939-44

68. Mironov SL. 2008. Metabotropic glutamate receptors activate dendritic calcium waves and TRPM channels which drive rhythmic respiratory patterns in mice. F. Physiol. 586:2277-91

69. Rekling JC, Shao XM, Feldman JL. 2000. Electrical coupling and excitatory synaptic transmission between rhythmogenic respiratory neurons in the preBötzinger Complex. F. Neurosci. 20:RC113

70. Krey RA, Goodreau AM, Arnold TB, Del Negro CA. 2010. Outward currents contributing to inspiratory burst termination in preBötzinger Complex neurons of neonatal mice studied in vitro. Front. Neural Circuits 4:124

71. Haller M, Mironov SL, Karschin A, Richter DW. 2001. Dynamic activation of $\mathrm{K}_{\mathrm{ATP}}$ channels in rhythmically active neurons. F. Physiol. 537:69-81

72. Mironov SL, Langohr K, Haller M, Richter DW. 1998. Hypoxia activates ATP-dependent potassium channels in inspiratory neurones of neonatal mice. 7. Physiol. 509(Pt. 3):755-66 
73. Mironov SL, Skorova EY. 2011. Stimulation of bursting in pre-Bötzinger neurons by Epac through calcium release and modulation of TRPM4 and K-ATP channels. 7. Neurochem. 117:295-308

74. Rall W, Burke RE, Smith TG, Nelson PG, Frank K. 1967. Dendritic location of synapses and possible mechanisms for the monosynaptic EPSP in motoneurons. F. Neurophysiol. 30:1169-93

75. Branco T, Hausser M. 2010. The single dendritic branch as a fundamental functional unit in the nervous system. Curr. Opin. Neurobiol. 20:494-502

76. Pace RW, Del Negro CA. 2008. AMPA and metabotropic glutamate receptors cooperatively generate inspiratory-like depolarization in mouse respiratory neurons in vitro. Eur. F. Neurosci. 28:2434-42

77. Pace RW, Mackay DD, Feldman JL, Del Negro CA. 2007. Inspiratory bursts in the preBötzinger Complex depend on a calcium-activated nonspecific cationic current linked to glutamate receptors. $\mathcal{F}$. Physiol. 582:113-25

78. Morgado-Valle C, Beltran-Parrazal L, DiFranco M, Vergara JL, Feldman JL. 2008. Somatic $\mathrm{Ca}^{2+}$ transients do not contribute to inspiratory drive in preBötzinger Complex neurons. F. Physiol. 586:453140

79. Crowder EA, Saha MS, Pace RW, Zhang H, Prestwich GD, Del Negro CA. 2007. Phosphatidylinositol 4,5-bisphosphate regulates inspiratory burst activity in the neonatal mouse preBötzinger Complex. $\mathcal{F}$. Physiol. 582:1047-58

80. London M, Hausser M. 2005. Dendritic computation. Annu. Rev. Neurosci. 28:503-32

81. Del Negro CA, Hayes JA, Rekling JC. 2011. Dendritic calcium activity precedes inspiratory bursts in preBötzinger Complex neurons. 7. Neurosci. 31:1017-22

82. Orlovsky GN, Deliagina TG, Grillner S. 1999. Neuronal Control of Locomotion: From Mollusc to Man. New York: Oxford Univ. Press. 322 pp.

83. Brown TG. 1914. On the nature of the fundamental activity of the nervous centres; together with an analysis of the conditioning of rhythmic activity in progression, and a theory of the evolution of function in the nervous system. 7. Physiol. 48:18-46

84. Sherrington CS. 1906. The Integrative Action of the Nervous System. New York: Yale Univ. Press. 411 pp.

85. Kiehn O. 2006. Locomotor circuits in the mammalian spinal cord. Annu. Rev. Neurosci. 29:279-306

86. Goulding M. 2009. Circuits controlling vertebrate locomotion: moving in a new direction. Nat. Rev. Neurosci. 10:507-18

87. Büsselberg D, Bischoff AM, Paton JF, Richter DW. 2001. Reorganisation of respiratory network activity after loss of glycinergic inhibition. Pflïg. Arch. 441:444-49

88. Büsselberg D, Bischoff AM, Richter DW. 2003. A combined blockade of glycine and calcium-dependent potassium channels abolishes the respiratory rhythm. Neuroscience 122:831-41

89. Richter DW, Spyer KM. 2001. Studying rhythmogenesis of breathing: comparison of in vivo and in vitro models. Trends Neurosci. 24:464-72

90. Molkov YI, Abdala AP, Bacak BJ, Smith JC, Paton JF, Rybak IA. 2010. Late-expiratory activity: emergence and interactions with the respiratory CPG. F. Neurophysiol. 104:2713-29

90a. Janczewski WA, Tashima A, Hsu P, Cui Y, Feldman JL. 2013. Role of inhibition in respiratory pattern generation. F. Neurosci. In press

91. Gray PA, Rekling JC, Bocchiaro CM, Feldman JL. 1999. Modulation of respiratory frequency by peptidergic input to rhythmogenic neurons in the preBötzinger Complex. Science 286:1566-68

92. Montandon G, Qin W, Liu H, Ren J, Greer JJ, Horner RL. 2011. PreBötzinger Complex neurokinin-1 receptor-expressing neurons mediate opioid-induced respiratory depression. F. Neurosci. 31:1292-301

93. Manzke T, Guenther U, Ponimaskin EG, Haller M, Dutschmann M, et al. 2003. 5-HT 4 (a) receptors avert opioid-induced breathing depression without loss of analgesia. Science 301:226-29

94. Bell SC, Saunders MJ, Elborn JS, Shale DJ. 1996. Resting energy expenditure and oxygen cost of breathing in patients with cystic fibrosis. Thorax 51:126-31

95. Gardner WN. 1980. The pattern of breathing following step changes of alveolar partial pressures of carbon dioxide and oxygen in man. 7. Physiol. 300:55-73

96. Aliverti A, Cala SJ, Duranti R, Ferrigno G, Kenyon CM, et al. 1997. Human respiratory muscle actions and control during exercise. 7. Appl. Physiol. 83:1256-69

97. Mellen NM, Janczewski WA, Bocchiaro CM, Feldman JL. 2003. Opioid-induced quantal slowing reveals dual networks for respiratory rhythm generation. Neuron 37:821-26 
98. Onimaru H, Homma I. 2003. A novel functional neuron group for respiratory rhythm generation in the ventral medulla. 7. Neurosci. 23:1478-86

99. Stornetta RL, Moreira TS, Takakura AC, Kang BJ, Chang DA, et al. 2006. Expression of Phox2b by brainstem neurons involved in chemosensory integration in the adult rat. $\mathcal{F}$. Neurosci. 26:1030514

100. Guyenet PG, Bayliss DA, Stornetta RL, Fortuna MG, Abbott SB, DePuy SD. 2009. Retrotrapezoid nucleus, respiratory chemosensitivity and breathing automaticity. Respir. Physiol. Neurobiol. 168:59-68

101. Smith JC, Morrison DE, Ellenberger HH, Otto MR, Feldman JL. 1989. Brainstem projections to the major respiratory neuron populations in the medulla of the cat. 7. Comp. Neurol. 281:69-96

102. Onimaru H, Homma I, Feldman JL, Janczewski WA. 2006. The parafacial respiratory group (pFRG)/pre-Bötzinger Complex (preBötC) is the primary site of respiratory rhythm generation in the mammal. 7. Appl. Physiol. 100:2094-98

103. Onimaru H, Homma I. 2008. Two modes of respiratory rhythm generation in the newborn rat brainstemspinal cord preparation. Adv. Exp. Med. Biol. 605:104-8

104. Fortuna MG, West GH, Stornetta RL, Guyenet PG. 2008. Bötzinger expiratory-augmenting neurons and the parafacial respiratory group. 7 . Neurosci. 28:2506-15

105. Mulkey DK, Talley EM, Stornetta RL, Siegel AR, West GH, et al. 2007. TASK channels determine pH sensitivity in select respiratory neurons but do not contribute to central respiratory chemosensitivity. 7. Neurosci. 27:14049-58

106. Pagliardini S, Janczewski WA, Tan W, Dickson CT, Deisseroth K, Feldman JL. 2011. Active expiration induced by excitation of ventral medulla in adult anesthetized rats. F. Neurosci. 31:2895-905

107. Eldridge FL, Millhorn DE, Kiley JP, Waldrop TG. 1985. Stimulation by central command of locomotion, respiration and circulation during exercise. Respir. Physiol. 59:313-37

108. de Almeida AT, Al-Izki S, Denton ME, Kirkwood PA. 2010. Patterns of expiratory and inspiratory activation for thoracic motoneurones in the anaesthetized and the decerebrate rat. $\mathcal{F}$. Physiol. 588:270729

109. de Almeida AT, Kirkwood PA. 2010. Multiple phases of excitation and inhibition in central respiratory drive potentials of thoracic motoneurones in the rat. F. Physiol. 588: 2731-44

110. Iizuka M. 2009. Abdominal expiratory muscle activity in anesthetized vagotomized neonatal rats. 7. Physiol. Sci. 59:157-63

111. Abdala AP, Rybak IA, Smith JC, Paton JF. 2009. Abdominal expiratory activity in the rat brainstem-spinal cord in situ: patterns, origins and implications for respiratory rhythm generation. F. Physiol. 587:3539-59

112. Iizuka M. 2004 Rostrocaudal distribution of spinal respiratory motor activity in an in vitro neonatal rat preparation. Neurosci. Res. 50:263-69

113. Taccola G, Secchia L, Ballanyi K. 2007. Anoxic persistence of lumbar respiratory bursts and block of lumbar locomotion in newborn rat brainstem spinal cords. 7. Physiol. 585:507-24

114. Cohen MI, Feldman JL. 1978. Central mechanisms controlling expiratory duration. Adv. Exp. Med. Biol. 99:369-82

115. Hayes JA, Del Negro CA. 2007. Neurokinin receptor-expressing pre-Bötzinger Complex neurons in neonatal mice studied in vitro. 7. Neurophysiol. 97:4215-24

116. Bogaard A, Parent J, Zochowski M, Booth V. 2009. Interaction of cellular and network mechanisms in spatiotemporal pattern formation in neuronal networks. F. Neurosci. 29:1677-87

117. Barabási A-L. 2003. Linked: How Everything Is Connected to Everything Else and What It Means for Business, Science, and Everyday Life. New York: Plume. 294 pp.

118. Hayes JA, Wang X, Del Negro CA. 2012. Cumulative lesioning of respiratory interneurons disrupts and precludes motor rhythms in vitro. Proc. Natl. Acad. Sci. USA 109:8286-91

119. Alheid GF, Milsom WK, McCrimmon DR. 2004. Pontine influences on breathing: an overview. Respir. Physiol. Neurobiol. 143:105-14

120. Bochorishvili G, Stornetta RL, Coates MB, Guyenet PG. 2012. Pre-Bötzinger Complex receives glutamatergic innervation from galaninergic and other retrotrapezoid nucleus neurons. 7. Comp. Neurol. 520:1047-61

121. Ma LH, Gilland E, Bass AH, Baker R. 2010. Ancestry of motor innervation to pectoral fin and forelimb. Nat. Commun. 1:49 
122. Straka H, Baker R, Gilland E. 2006. Preservation of segmental hindbrain organization in adult frogs. 7. Comp. Neurol. 494:228-45

123. Gray PA. 2008. Transcription factors and the genetic organization of brain stem respiratory neurons. 7. Appl. Physiol. 104:1513-21

124. Gray PA, Fu H, Luo P, Zhao Q, Yu J, et al. 2004. Mouse brain organization revealed through direct genome-scale TF expression analysis. Science 306:2255-57

125. Briscoe J, Pierani A, Jessell TM, Ericson J. 2000. A homeodomain protein code specifies progenitor cell identity and neuronal fate in the ventral neural tube. Cell 101:435-45

126. Lumsden A. 1990. The cellular basis of segmentation in the developing hindbrain. Trends Neurosci. $13: 329-35$

127. Sieber MA, Storm R, Martinez-de-la-Torre M, Muller T, Wende H, et al. 2007. Lbx1 acts as a selector gene in the fate determination of somatosensory and viscerosensory relay neurons in the hindbrain. 7. Neurosci. 27:4902-9

128. Guyenet PG, Sevigny CP, Weston MC, Stornetta RL. 2002. Neurokinin-1 receptor-expressing cells of the ventral respiratory group are functionally heterogeneous and predominantly glutamatergic. 7. Neurosci. 22:3806-16

129. Guyenet PG, Wang H. 2001. Pre-Bötzinger neurons with preinspiratory discharges "in vivo" express NK1 receptors in the rat. 7. Neurophysiol. 86:438-46

130. Wang H, Stornetta RL, Rosin DL, Guyenet PG. 2001. Neurokinin-1 receptor-immunoreactive neurons of the ventral respiratory group in the rat. 7. Comp. Neurol. 434:128-46

131. Tan W, Sherman D, Turesson J, Shao XM, Janczewski WA, Feldman JL. 2012. Reelin demarks a subset of pre-Bötzinger Complex neurons in adult rat. F. Comp. Neurol. 520:606-19

132. Gray PA, Hayes JA, Ling GY, Llona I, Tupal S, et al. 2010. Developmental origin of preBötzinger Complex respiratory neurons. 7. Neurosci. 30:14883-95

133. Shvarev Y, Berner J, Bilkei-Gorzo A, Lagercrantz H, Wickstrom R. 2010. Acute morphine effects on respiratory activity in mice with target deletion of the tachykinin 1 gene (Tac1-/- ). Adv. Exp. Med. Biol. 669:129-32

134. Doi A, Ramirez JM. 2008. Neuromodulation and the orchestration of the respiratory rhythm. Respir. Physiol. Neurobiol. 164:96-104

135. Berner J, Shvarev Y, Lagercrantz H, Bilkei-Gorzo A, Hokfelt T, Wickstrom R. 2007. Altered respiratory pattern and hypoxic response in transgenic newborn mice lacking the tachykinin-1 gene. $\mathcal{F}$. Appl. Physiol. 103:552-59

136. Hilaire G, Burnet H, Ptak K, Sieweke M, Blanchi B, et al. 2003. Deletion of tachykinin NK1 receptor gene in mice does not alter respiratory network maturation but alters respiratory responses to hypoxia. Adv. Exp. Med. Biol. 536:497-504

137. Schreihofer AM, Stornetta RL, Guyenet PG. 1999. Evidence for glycinergic respiratory neurons: Bötzinger neurons express mRNA for glycinergic transporter 2. 7. Comp. Neurol. 407:583-97

138. Winter SM, Fresemann J, Schnell C, Oku Y, Hirrlinger J, Hulsmann S. 2010. Glycinergic interneurons in the respiratory network of the rhythmic slice preparation. Adv. Exp. Med. Biol. 669:97-100

139. Greer JJ, Smith JC, Feldman JL. 1991. Role of excitatory amino acids in the generation and transmission of respiratory drive in neonatal rat. 7. Physiol. 437:727-49

140. Blanchi B, Sieweke MH. 2005. Mutations of brainstem transcription factors and central respiratory disorders. Trends Mol. Med. 11:23-30

141. Rose MF, Ren J, Ahmad KA, Chao HT, Klisch TJ, et al. 2009. Math1 is essential for the development of hindbrain neurons critical for perinatal breathing. Neuron 64:341-54

142. Blanchi B, Kelly LM, Viemari JC, Lafon I, Burnet H, et al. 2003. MafB deficiency causes defective respiratory rhythmogenesis and fatal central apnea at birth. Nat. Neurosci. 6:1091-100

143. Pagliardini S, Ren J, Gray PA, Vandunk C, Gross M, et al. 2008. Central respiratory rhythmogenesis is abnormal in Lbx1- deficient mice. 7. Neurosci. 28:11030-41

144. Borday C, Chatonnet F, Thoby-Brisson M, Champagnat J, Fortin G. 2005. Neural tube patterning by Krox20 and emergence of a respiratory control. Respir. Physiol. Neurobiol. 149:63-72

145. Thoby-Brisson M, Karlen M, Wu N, Charnay P, Champagnat J, Fortin G. 2009. Genetic identification of an embryonic parafacial oscillator coupling to the preBötzinger Complex. Nat. Neurosci. 12:1028-35 
146. Nsegbe E, Wallen-Mackenzie A, Dauger S, Roux JC, Shvarev Y, et al. 2004. Congenital hypoventilation and impaired hypoxic response in Nurr1 mutant mice. 7. Physiol. 556:43-59

147. Dubreuil V, Ramanantsoa N, Trochet D, Vaubourg V, Amiel J, et al. 2008. A human mutation in Pbox $2 b$ causes lack of $\mathrm{CO}_{2}$ chemosensitivity, fatal central apnea, and specific loss of parafacial neurons. Proc. Natl. Acad. Sci. USA 105:1067-72

148. Pattyn A, Goridis C, Brunet JF. 2000. Specification of the central noradrenergic phenotype by the homeobox gene Phox2b. Mol. Cell. Neurosci. 15:235-43

149. Caubit X, Thoby-Brisson M, Voituron N, Filippi P, Bevengut M, et al. 2010. Teashirt 3 regulates development of neurons involved in both respiratory rhythm and airflow control. 7. Neurosci. 30:946576

150. Glasgow SM, Henke RM, Macdonald RJ, Wright CV, Johnson JE. 2005. Ptf1a determines GABAergic over glutamatergic neuronal cell fate in the spinal cord dorsal horn. Development 132:5461-69

151. Goridis C, Dubreuil V, Thoby-Brisson M, Fortin G, Brunet JF. 2010. Phox2b, congenital central hypoventilation syndrome and the control of respiration. Semin. Cell Dev. Biol. 21:814-22

152. Cheng L, Arata A, Mizuguchi R, Qian Y, Karunaratne A, et al. 2004. Tlx3 and Tlx1 are post-mitotic selector genes determining glutamatergic over GABAergic cell fates. Nat. Neurosci. 7:510-17

153. Rose MF, Ahmad KA, Thaller C, Zoghbi HY. 2009. Excitatory neurons of the proprioceptive, interoceptive, and arousal hindbrain networks share a developmental requirement for Math1. Proc. Natl. Acad. Sci. USA 106:22462-67

154. Pierani A, Moran-Rivard L, Sunshine MJ, Littman DR, Goulding M, Jessell TM. 2001. Control of interneuron fate in the developing spinal cord by the progenitor homeodomain protein Dbx1. Neuron 29:367-84

155. Lanuza GM, Gosgnach S, Pierani A, Jessell TM, Goulding M. 2004. Genetic identification of spinal interneurons that coordinate left-right locomotor activity necessary for walking movements. Neuron 42:375-86

156. Bouvier J, Thoby-Brisson M, Renier N, Dubreuil V, Ericson J, et al. 2010. Hindbrain interneurons and axon guidance signaling critical for breathing. Nat. Neurosci. 13:1066-74

157. Gosgnach S. 2011. The role of genetically-defined interneurons in generating the mammalian locomotor rhythm. Integ. Comp. Biol. 51:903-12

158. Champagnat J, Morin-Surun MP, Fortin G, Thoby-Brisson M. 2009. Developmental basis of the rostro-caudal organization of the brainstem respiratory rhythm generator. Philos. Trans. R. Soc. Lond. B 364:2469-76

159. Neumueller S, Hodges MR, Krause K, Marshall B, Bonis J, et al. 2011. Anatomic changes in multiple brainstem nuclei after incremental, near-complete neurotoxic destruction of the pre-Bötzinger Complex in adult goats. Respir. Physiol. Neurobiol. 175:1-11

160. Krause KL, Forster HV, Kiner T, Davis SE, Bonis JM, et al. 2009. Normal breathing pattern and arterial blood gases in awake and sleeping goats after near total destruction of the presumed pre-Bötzinger Complex and the surrounding region. F. Appl. Physiol. 106:605-19

161. Wenninger JM, Pan LG, Klum L, Leekley T, Bastastic J, et al. 2004. Large lesions in the pre-Bötzinger Complex area eliminate eupneic respiratory rhythm in awake goats. F. Appl. Physiol. 97:1629-36

162. Wenninger JM, Pan LG, Klum L, Leekley T, Bastastic J, et al. 2004. Small reduction of neurokinin-1 receptor-expressing neurons in the pre-Bötzinger Complex area induces abnormal breathing periods in awake goats. F. Appl. Physiol. 97:1620-28

163. Wang H, Weston MC, McQuiston TJ, Stornetta RL, Guyenet PG. 2003. Neurokinin-1 receptorexpressing cells regulate depressor region of rat ventrolateral medulla. Am. 7. Physiol. Heart Circ. Physiol. 285:H2757-69

164. Weese-Mayer DE, Berry-Kravis EM, Ceccherini I, Keens TG, Loghmanee DA, Trang H. 2010. An official ATS clinical policy statement: congenital central hypoventilation syndrome: genetic basis, diagnosis, and management. Am. 7. Respir. Crit. Care Med. 181:626-44

165. Takakura AC, Moreira TS, Stornetta RL, West GH, Gwilt JM, Guyenet PG. 2008. Selective lesion of retrotrapezoid Phox2b-expressing neurons raises the apnoeic threshold in rats. F. Physiol. 586:2975-91 
166. Abbott SB, Stornetta RL, Fortuna MG, Depuy SD, West GH, et al. 2009. Photostimulation of retrotrapezoid nucleus Phox2b-expressing neurons in vivo produces long-lasting activation of breathing in rats. 7. Neurosci. 29:5806-19

167. Abbott SB, Stornetta RL, Coates MB, Guyenet PG. 2011. Phox2b-expressing neurons of the parafacial region regulate breathing rate, inspiration, and expiration in conscious rats. F. Neurosci. 31:1641022

168. Guyenet PG, Mulkey DK, Stornetta RL, Bayliss DA. 2005. Regulation of ventral surface chemoreceptors by the central respiratory pattern generator. 7. Neurosci. 25:8938-47

169. Mulkey DK, Stornetta RL, Weston MC, Simmons JR, Parker A, et al. 2004. Respiratory control by ventral surface chemoreceptor neurons in rats. Nat. Neurosci. 7:1360-69

170. Kang BJ, Chang DA, Mackay DD, West GH, Moreira TS, et al. 2007. Central nervous system distribution of the transcription factor Phox2b in the adult rat. 7. Comp. Neurol. 503:627-41

171. Pattyn A, Morin X, Cremer H, Goridis C, Brunet JF. 1997. Expression and interactions of the two closely related homeobox genes Phox $2 a$ and Phox $2 b$ during neurogenesis. Development 124:4065-75

172. Jacquin TD, Borday V, Schneider-Maunoury S, Topilko P, Ghilini G, et al. 1996. Reorganization of pontine rhythmogenic neuronal networks in Krox-20 knockout mice. Neuron 17:747-58

173. Ramanantsoa N, Hirsch MR, Thoby-Brisson M, Dubreuil V, Bouvier J, et al. 2011. Breathing without $\mathrm{CO}_{2}$ chemosensitivity in conditional Phox $2 b$ mutants. F. Neurosci. 31:12880-88

174. Huang WH, Tupal S, Huang TW, Ward CS, Neul JL, et al. 2012. Atoh1 governs the migration of postmitotic neurons that shape respiratory effectiveness at birth and chemoresponsiveness in adulthood. Neuron 75:799-809

175. Thoby-Brisson M, Trinh JB, ChampagnatJ, Fortin G. 2005. Emergence of the pre-Bötzinger respiratory rhythm generator in the mouse embryo. F. Neurosci. 25:4307-18

176. Onimaru H, Ikeda K, Kawakami K. 2008. $\mathrm{CO}_{2}$-sensitive preinspiratory neurons of the parafacial respiratory group express Phox $2 \mathrm{~b}$ in the neonatal rat. F. Neurosci. 28:12845-50

177. Onimaru H, Ikeda K, Kawakami K. 2012. Postsynaptic mechanisms of $\mathrm{CO}_{2}$ responses in parafacial respiratory neurons of newborn rats. F. Physiol. 590:1615-24

178. Vasilakos K, Wilson RJ, Kimura N, Remmers JE. 2005. Ancient gill and lung oscillators may generate the respiratory rhythm of frogs and rats. F. Neurobiol. 62:369-85

179. Hodges MR, Richerson GB. 2010. Medullary serotonin neurons and their roles in central respiratory chemoreception. Respir. Physiol. Neurobiol. 173:256-63

180. Hendricks TJ, Fyodorov DV, Wegman LJ, Lelutiu NB, Pehek EA, et al. 2003. Pet-1 ETS gene plays a critical role in 5-HT neuron development and is required for normal anxiety-like and aggressive behavior. Neuron 37:233-47

181. Hodges MR, Tattersall GJ, Harris MB, McEvoy SD, Richerson DN, et al. 2008. Defects in breathing and thermoregulation in mice with near-complete absence of central serotonin neurons. 7 . Neurosci. 28:2495-505

182. Hodges MR, Best S, Richerson GB. 2011. Altered ventilatory and thermoregulatory control in male and female adult Pet-1 null mice. Respir. Physiol. Neurobiol. 177:133-40

183. Hodges MR, Wehner M, Aungst J, Smith JC, Richerson GB. 2009. Transgenic mice lacking serotonin neurons have severe apnea and high mortality during development. 7 . Neurosci. 29:10341-49

184. Ray RS, Corcoran AE, Brust RD, Kim JC, Richerson GB, et al. 2011. Impaired respiratory and body temperature control upon acute serotonergic neuron inhibition. Science 333:637-42

185. Huckstepp RT, Dale N. 2011. Redefining the components of central $\mathrm{CO}_{2}$ chemosensitivity-towards a better understanding of mechanism. F. Physiol. 589:5561-79

186. Richter DW, Manzke T, Wilken B, Ponimaskin E. 2003. Serotonin receptors: guardians of stable breathing. Trends Mol. Med. 9:542-48

187. Doi A, Ramirez JM. 2010. State-dependent interactions between excitatory neuromodulators in the neuronal control of breathing. F. Neurosci. 30:8251-62

188. Paterson DS, Trachtenberg FL, Thompson EG, Belliveau RA, Beggs AH, et al. 2006. Multiple serotonergic brainstem abnormalities in sudden infant death syndrome. FAMA 296:2124-32

189. Peña F, Ramirez JM. 2002. Endogenous activation of serotonin-2A receptors is required for respiratory rhythm generation in vitro. 7. Neurosci. 22:11055-64 
190. Lu B, Su Y, Das S, Liu J, Xia J, Ren D. 2007. The neuronal channel NALCN contributes resting sodium permeability and is required for normal respiratory rhythm. Cell 129:371-83

191. Gourine AV, Kasymov V, Marina N, Tang F, Figueiredo MF, et al. 2010. Astrocytes control breathing through $\mathrm{pH}$-dependent release of ATP. Science 329:571-75

192. Nattie EE, Li A. 1996. Central chemoreception in the region of the ventral respiratory group in the rat. 7. Appl. Physiol. 81:1987-95

193. Schlafke ME, Pokorski M, See WR, Prill RK, Loeschcke HH. 1975. Chemosensitive neurons on the ventral medullary surface. Bull. Physiopathol. Respir. 11:277-84

194. Gourine AV, Llaudet E, Dale N, Spyer KM. 2005. ATP is a mediator of chemosensory transduction in the central nervous system. Nature 436:108-11

195. Huckstepp RT, Eason R, Sachdev A, Dale N. 2010. $\mathrm{CO}_{2}$-dependent opening of connexin 26 and related beta connexins. F. Physiol. 588:3921-31

196. Huckstepp RT, id Bihi R, Eason R, Spyer KM, Dicke N, et al. 2010. Connexin hemichannel-mediated $\mathrm{CO}_{2}$-dependent release of ATP in the medulla oblongata contributes to central respiratory chemosensitivity. F. Physiol. 588:3901-20

197. Wenker IC, Sobrinho CR, Takakura AC, Moreira TS, Mulkey DK. 2012. Regulation of ventral surface $\mathrm{CO}_{2} / \mathrm{H}^{+}$-sensitive neurons by purinergic signaling. 7. Physiol. 590(Pt. 9):2137-50

198. Weese-Mayer DE, Berry-Kravis EM, Ceccherini I, Rand CM. 2008. Congenital central hypoventilation syndrome (CCHS) and sudden infant death syndrome (SIDS): kindred disorders of autonomic regulation. Respir. Physiol. Neurobiol. 164:38-48

199. Weese-Mayer DE, Lieske SP, Boothby CM, Kenny AS, Bennett HL, et al. 2006. Autonomic nervous system dysregulation: breathing and heart rate perturbation during wakefulness in young girls with Rett syndrome. Pediatr. Res. 60:443-49

200. Ben-Shachar S, Chahrour M, Thaller C, Shaw CA, Zoghbi HY. 2009. Mouse models of MeCP2 disorders share gene expression changes in the cerebellum and hypothalamus. Hum. Mol. Genet. 18:2431-42

201. Valente EM, Silhavy JL, Brancati F, Barrano G, Krishnaswami SR, et al. 2006. Mutations in CEP290, which encodes a centrosomal protein, cause pleiotropic forms of Joubert syndrome. Nat. Genet. 38:62325

202. Valente EM, Brancati F, Silhavy JL, Castori M, Marsh SE, et al. 2006. AHI1 gene mutations cause specific forms of Joubert syndrome-related disorders. Ann. Neurol. 59:527-34

203. Weese-Mayer DE, Lieske SP, Boothby CM, Kenny AS, Bennett HL, Ramirez JM. 2008. Autonomic dysregulation in young girls with Rett syndrome during nighttime in-home recordings. Pediatr. Pulmonol. 43:1045-60

204. Dunn HG, MacLeod PM. 2001. Rett syndrome: review of biological abnormalities. Can. F. Neurol. Sci. 28:16-29

205. Voituron N, Zanella S, Menuet C, Lajard AM, Dutschmann M, Hilaire G. 2010. Early abnormalities of post-sigh breathing in a mouse model of Rett syndrome. Respir. Physiol. Neurobiol. 170:17382

206. Viemari JC, Roux JC, Tryba AK, Saywell V, Burnet H, et al. 2005. Mecp2 deficiency disrupts norepinephrine and respiratory systems in mice. 7. Neurosci. 25:11521-30

207. Lioy DT, Garg SK, Monaghan CE, Raber J, Foust KD, et al. 2011. A role for glia in the progression of Rett's syndrome. Nature 475:497-500

208. Robinson L, Guy J, McKay L, Brockett E, Spike RC, et al. 2012. Morphological and functional reversal of phenotypes in a mouse model of Rett syndrome. Brain 135:2699-710

209. Benarroch EE. 2003. Brainstem in multiple system atrophy: clinicopathological correlations. Cell. Mol. Neurobiol. 23:519-26

210. Benarroch EE. 2007. Brainstem respiratory control: substrates of respiratory failure of multiple system atrophy. Mov. Disord. 22:155-61

211. Benarroch EE, Schmeichel AM, Low PA, Parisi JE. 2003. Depletion of ventromedullary NK-1 receptorimmunoreactive neurons in multiple system atrophy. Brain 126:2183-90

212. Schwarzacher SW, Rüb U, Deller T. 2011. Neuroanatomical characteristics of the human pre-Bötzinger Complex and its involvement in neurodegenerative brainstem diseases. Brain 134(Pt. 1):24-35 
213. Rand CM, Patwari PP, Rodikova EA, Zhou L, Berry-Kravis EM, et al. 2011. Rapid-onset obesity with hypothalamic dysfunction, hypoventilation, and autonomic dysregulation: analysis of hypothalamic and autonomic candidate genes. Pediatr. Res. 70:375-78

214. Ize-Ludlow D, Gray JA, Sperling MA, Berry-Kravis EM, Milunsky JM, et al. 2007. Rapid-onset obesity with hypothalamic dysfunction, hypoventilation, and autonomic dysregulation presenting in childhood. Pediatrics 120:e179-88

215. Weese-Mayer DE. 2008. Sudden infant death syndrome: the genetic segue? Acta Paediatr. 97:846-47

216. Paterson DS, Hilaire G, Weese-Mayer DE. 2009. Medullary serotonin defects and respiratory dysfunction in sudden infant death syndrome. Respir. Physiol. Neurobiol. 168:133-43

217. Tsuboi Y, Dickson DW, Nabeshima K, Schmeichel AM, Wszolek ZK, et al. 2008. Neurodegeneration involving putative respiratory neurons in Perry syndrome. Acta Neuropathol. 115:263-68 
Annual Review of Physiology

Volume 75, 2013

\section{Contents}

PERSPECTIVES, David Fulius, Editor

A Conversation with Roger Guillemin

Roger Guillemin and Greg Lemke 1

CARDIOVASCULAR PHYSIOLOGY, Marlene Rabinovitch, Section Editor

The Adventitia: Essential Regulator of Vascular Wall Structure and Function

Kurt R. Stenmark, Michael E. Yeager, Karim C. El Kasmi, Eva Nozik-Grayck, Evgenia V. Gerasimovskaya, Min Li, Suzette R. Riddle, and Maria G. Frid

Coping with Endoplasmic Reticulum Stress in the Cardiovascular System

Fody Groenendyk, Luis B. Agellon, and Marek Michalak

Functions of MicroRNAs in Cardiovascular Biology and Disease Akiko Hata

Mitochondria in Vascular Health and Disease

Peter Dromparis and Evangelos D. Michelakis

CELL PHYSIOLOGY, David E. Clapham, Section Editor

Mammalian Phospholipase C

Ganesh Kadamur and Elliott M. Ross

Regulation of Insulin Secretion in Human Pancreatic Islets

Patrik Rorsman and Matthias Braun

TRPA1: A Gatekeeper for Inflammation

Diana M. Bautista, Maurizio Pellegrino, and Makoto Tsunozaki 181 
ENDOCRINOLOGY, Holly A. Ingraham, Section Editor

The Androgen Receptor in Health and Disease

Takabiro Matsumoto, Matomo Sakari, Maiko Okada, Atsushi Yokoyama, Sayuri Takahashi, Alexander Kouzmenko, and Shigeaki Kato

Estrogen and Cancer

Fing Liang and Yongfeng Shang

GASTROINTESTINAL PHYSIOLOGY, Linda Samuelson, Section Editor

Autophagy and Intestinal Homeostasis

Khushbu K. Patel and Thaddeus S. Stappenbeck

Notch in the Intestine: Regulation of Homeostasis and Pathogenesis

Taeko K. Noab and Noab F. Shroyer

Paneth Cells: Maestros of the Small Intestinal Crypts

Hans C. Clevers and Charles L. Bevins

NEUROPHYSIOLOGY, Roger Nicoll, Section Editor

Functional Insights from Glutamate Receptor Ion Channel Structures fanesh Kumar and Mark L. Mayer

The Impact of Adult Neurogenesis on Olfactory Bulb Circuits and Computations

Gabriel Lepousez, Matthew T. Valley, and Pierre-Marie Lledo

Pathophysiology of Migraine

Daniela Pietrobon and Michael A. Moskowitz

Perspectives on Kiss-and-Run: Role in Exocytosis, Endocytosis, and Neurotransmission AbdulRasheed A. Alabi and Richard W. Tsien

Understanding the Rhythm of Breathing: So Near, Yet So Far Jack L. Feldman, Christopher A. Del Negro, and Paul A. Gray

RENAL AND ELECTROLYTE PHYSIOLOGY, Peter Aronson, Section Editor

Chloride in Vesicular Trafficking and Function

Tobias Stauber and Thomas 7. Fentsch 453

Claudins and the Kidney

Fianghui Hou, Madhumitha Rajagopal, and Alan S.L. Yu

Fibroblast Growth Factor 23 and Klotho: Physiology and

Pathophysiology of an Endocrine Network of Mineral Metabolism

Ming Chang Hu, Kazuhiro Shiizaki, Makoto Kuro-o, and Orson W. Moe 
Phosphate Transporters and Their Function fürg Biber, Nati Hernando, and Ian Forster

RESPIRATORY PHYSIOLOGY, Augustine M.K. Choi, Section Editor

Claudin Heterogeneity and Control of Lung Tight Junctions Michael Koval

Platelets in Lung Biology

Andrew S. Weyrich and Guy A. Zimmerman

Regulation and Repair of the Alveolar-Capillary Barrier in Acute Lung Injury Jahar Bhattacharya and Michael A. Matthay

SPECIAL TOPIC, AGING AND LONGEVITY, Thomas A. Rando, Section Editor

The Ins and Outs of Aging and Longevity Thomas A. Rando

Genetics of Longevity in Model Organisms: Debates and Paradigm Shifts David Gems and Linda Partridge

Genome Instability and Aging Fan Vijg and Yousin Sub

Metabolic and Neuropsychiatric Effects of Calorie Restriction and Sirtuins Sergiy Libert and Leonard Guarente

Aging, Cellular Senescence, and Cancer Fudith Campisi

\section{Indexes}

Cumulative Index of Contributing Authors, Volumes 71-75 707

Cumulative Index of Article Titles, Volumes 71-75

\section{Errata}

An online log of corrections to Annual Review of Physiology articles may be found at http://physiol.annualreviews.org/errata.shtml 